\title{
Diameters of Cocircuit Graphs of Oriented Matroids: An Update
}

\author{
Ilan Ader \\ Univ. of California, Berkeley \\ California, U.S.A. \\ adler@ieor. berkeley.edu \\ Steven Klee \\ Department of Mathematics \\ Seattle University \\ Washington, U.S.A. \\ klees@seattleu.edu
}

Dept. of Industrial Engineering and Operations Research

\author{
Jesús A. De Loera \\ Department of Mathematics \\ Univ. of California, Davis \\ California, U.S.A. \\ deloera@math.ucdavis.edu
}

\author{
Zhenyang Zhang \\ Department of Mathematics \\ Univ. of California, Davis \\ California, U.S.A. \\ zhenyangz@math.ucdavis.edu
}

Submitted: Jun 16, 2020; Accepted: Nov 9, 2021; Published: Dec 3, 2021

(C) The authors. Released under the CC BY-ND license (International 4.0).

\begin{abstract}
Oriented matroids are combinatorial structures that generalize point configurations, vector configurations, hyperplane arrangements, polyhedra, linear programs, and directed graphs. Oriented matroids have played a key role in combinatorics, computational geometry, and optimization. This paper surveys prior work and presents an update on the search for bounds on the diameter of the cocircuit graph of an oriented matroid. The motivation for our investigations is the complexity of the simplex method and the criss-cross method.

We review the diameter problem and show the diameter bounds of general oriented matroids reduce to those of uniform oriented matroids. We give the latest exact bounds for oriented matroids of low rank and low corank, and for all oriented matroids with up to nine elements (this part required a large computer-based proof). For arbitrary oriented matroids, we present an improvement to a quadratic bound of Finschi. Our discussion highlights an old conjecture that states a linear bound for the diameter is possible. On the positive side, we show the conjecture is true for oriented matroids of low rank and low corank, and, verified with computers, for all oriented matroids with up to nine elements. On the negative side, our computer search showed two natural strengthenings of the main conjecture are false.
\end{abstract}

Mathematics Subject Classifications: 52C40, 05C12, 52C45, 52B40 


\section{Introduction}

Oriented matroids are combinatorial structures that generalize many types of objects, including point configurations, vector configurations, hyperplane arrangements, polyhedra, linear programs, and directed graphs. Oriented matroids have played a key role in combinatorics, geometry, and optimization (see the book by Björner et al. [4] for a complete treatise and Chapter 6 of Ziegler [29] for a quick introduction we assume in the rest). In this paper we investigate a natural graph called the cocircuit graph of an oriented matroid.

An important family of oriented matroids, realizable oriented matroids, are given by hyperplane arrangements. In this case, the cocircuit graph is just the one-skeleton of the cell complex obtained by intersecting a central hyperplane arrangement with a unit sphere. In general, the cocircuit graph is the graph of a combinatorial manifold and it has a rich structure. Later we review this geometry in some detail.

General characterizations and properties of oriented matroids and their cocircuit graphs have been explored by several authors: While it is known that the cocircuit graph does not uniquely determine the oriented matroid (see $[5,6]$ ), labeled cocircuit graphs can be characterized (see $[2,6]$ ). Other topics of research have been the connectivity of the cocircuit graph (see $[5,4]$ ) and how the cocircuit graph could define the entire oriented matroid and discussed the connectivity of the graph (see $[11,21,23]$ ). In this article we are interested instead in bounding the diameter of the cocircuit graph of an oriented matroid. We recall that the diameter of a graph is the largest distance between a pair of its vertices, where the distance between two vertices is the length of a shortest path connecting them.

The motivation for our investigations is the complexity of the simplex method $[3,27]$ and of the criss-cross method [16, 17]. Both algorithms are pivoting methods that jump from cocircuit to cocircuit along edges of the cocircuit graph. Bounds on the diameter are relevant for understanding their running time. The following conjecture is the oldest and the most ambitious open challenge about the diameter of oriented matroids today.

Conjecture 1. Let $\mathcal{M}$ be an oriented matroid of rank $r$ on $n$ elements, and let $G^{*}(\mathcal{M})$ be its cocircuit graph. Then $\operatorname{diam}\left(G^{*}(\mathcal{M})\right) \leqslant n-r+2$.

Prof. K. Fukuda (personal communication) kindly informed us that Conjecture 1 is an old folklore problem that goes back at least 25 years. We hope to revive interest in this conjecture with this article. Conjecture 1 bears a striking resemblance to the famous Hirsch conjecture for convex polytopes, which was disproved by F. Santos in [25], and with good reason. Let $P \subseteq \mathbb{R}^{d}$ be a $d$-polytope defined by $n$ hyperplane inequalities. Lifting $P$ to $\mathbb{R}^{d+1}$ (and setting $r=d+1$ ) determines a central hyperplane arrangement in $\mathbb{R}^{r}$, one of whose cones is the nonnegative span of $P$. Therefore, $P$ gives rise to an oriented matroid $\mathcal{M}$ whose cocircuit graph contains the graph of $P$ as an induced subgraph (see Figure 1).

Substituting $r=d+1$ in Conjecture 1 gives an upper bound of $n-r+2=n-d+1$, which differs from the conjectured Hirsch bound by 1 . The reason for this is that each signed cocircuit $X$ has an antipodal cocircuit $-X$. We will see later that when $\mathcal{M}$ is uniform, the distance between antipodal cocircuits is exactly $n-r+2$. 

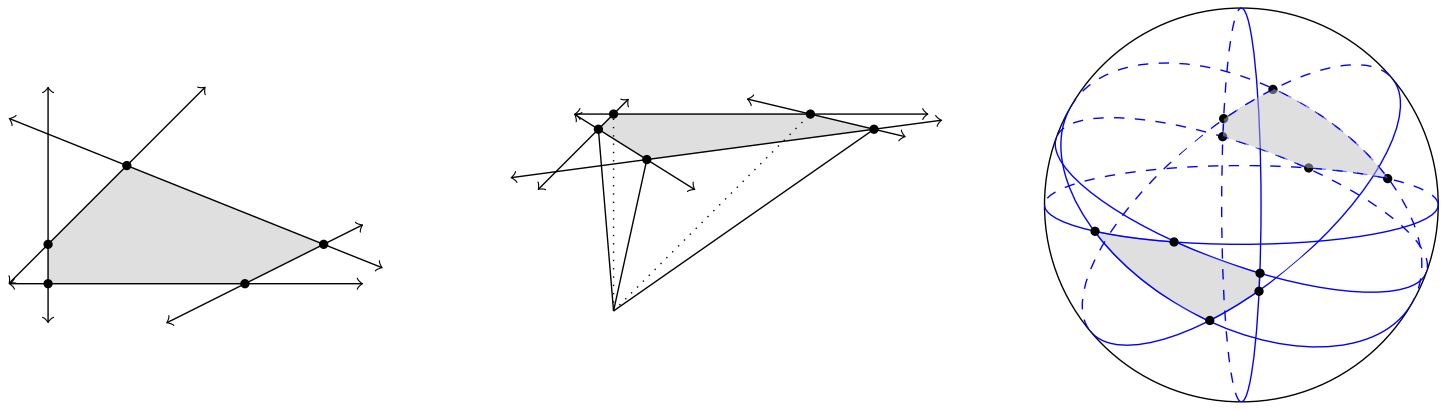

Figure 1: A polytope in $\mathbb{R}^{2}$ (left), its lifting to $\mathbb{R}^{3}$ (center), and the intersection with the resulting hyperplane arrangement on $\mathbb{S}^{2}$ (right).

Conjecture 1 has appeared in the literature in several forms. Babson, Finschi, and Fukuda [2, Lemma 6] established Conjecture 1 for uniform oriented matroids of rank 2 and rank 3 , showing further that only antipodal cocircuits can have distance $n-r+2$. Felsner et al. [11, Lemma 4.1] also showed that the conjecture is true for uniform oriented matroids with rank at most 3 and stated again the famous Conjecture 1 in [11, Question 4.2] with a strong emphasis on the important role of antipodal cocircuits. Finschi [12, Open Problem $5]$ asked whether $\operatorname{diam}\left(G^{*}(\mathcal{M})\right) \leqslant c \cdot n$ for some constant $c$ that is independent of $n$ and $r$. Aside from the results of Babson, Finschi, and Fukuda in low rank, the most general progress that has been made on Conjecture 1 seems to have come from Finschi's Ph.D thesis.

Theorem 2. (Finschi [12, Proposition 2.6.1])

Let $\mathcal{M}$ be a uniform oriented matroid of rank $r$ on $n$ elements. Then

$$
\operatorname{diam}\left(G^{*}(\mathcal{M})\right) \leqslant n-r+2+\sum_{k=1}^{\min (r-2, n-r)}\left(\left\lfloor\frac{n-r-k}{2}\right\rfloor+1\right) .
$$

The bound in Theorem 2 is tight when $r=2$ or $r=n$, but in general it is not.

\section{$1.1 \quad$ Notation and Definitions}

We use standard notation about oriented matroids from Ziegler [29] and the classic book of Björner et al. [4]. The geometric intuition that accompanies these definitions will be discussed at greater length in Section 2, but for now we introduce the minimal notation and definitions so that we can state our results formally.

A purely combinatorial description of oriented matroids can be given in terms of special sign vectors. If $E$ is a finite set, we use $\{+,-, 0\}^{E}$ to denote the set of all vectors of signs, with entries indexed by the elements of $E$. We will use capital letters $X, Y, Z, \ldots$ to represent elements of $\{+,-, 0\}^{E}$ and subscripts $X_{e}$ to reference the entry of $X$ indexed by the element $e \in E$. We can always negate a sign vector: if $X=\left(X_{e}: e \in E\right)$, then $-X=\left(-X_{e}: e \in E\right)$. 
The positive, negative, and zero parts of a sign vector $X \in\{+,-, 0\}^{E}$ are defined respectively as $X^{+}=\left\{e \in E: X_{e}=+\right\}, X^{-}=\left\{e \in E: X_{e}=-\right\}$, and $X^{0}=\{e \in E$ : $\left.X_{e}=0\right\}$. The support of $X$ is defined as $\operatorname{supp}(X)=X^{+} \cup X^{-}$. If $X$ and $Y$ are sign vectors, their separating set is $S(X, Y)=\left(X^{+} \cap Y^{-}\right) \cup\left(X^{-} \cap Y^{+}\right)$, and their composition is the sign vector $X \circ Y$ whose entries are given by

$$
(X \circ Y)_{e}= \begin{cases}X_{e} & \text { if } X_{e} \neq 0 \\ Y_{e} & \text { otherwise }\end{cases}
$$

For the moment we will only provide the cocircuit axioms of oriented matroids. As with classical matroids, there are also several cryptomorphic definitions of oriented matroids; see [4] for more details. We will briefly introduce some of these details later. The cocircuits and covectors of an oriented matroid are special types of sign vectors that satisfy certain axioms:

Definition 3. An oriented matroid $\mathcal{M}=\left(E, \mathcal{C}^{*}\right)$ consists of a finite set $E$ and a subset $\mathcal{C}^{*} \subseteq\{+,-, 0\}^{E}$, called signed cocircuits, that satisfy the following conditions.

$(\mathrm{CC} 0) \mathbf{0} \notin \mathcal{C}^{*}$;

$(\mathrm{CC} 1)$ if $X \in \mathcal{C}^{*}$, then $-X \in \mathcal{C}^{*}$;

(CC2) for all $X, Y \in \mathcal{C}^{*}$, if $\operatorname{supp}(X) \subseteq \operatorname{supp}(Y)$, then $X=Y$ or $X=-Y$; and

(CC3) if $X, Y \in \mathcal{C}^{*}, X \neq-Y$, and $e \in S(X, Y)$, then there exists $Z \in \mathcal{C}^{*}$ such that $Z^{+} \subseteq\left(X^{+} \cup Y^{+}\right) \backslash\{e\}$ and $Z^{-} \subseteq\left(X^{-} \cup Y^{-}\right) \backslash\{e\}$.

Given an oriented matroid $\mathcal{M}$, we can consider the set $\mathcal{V}^{*}=\left\{X^{0} \circ X^{1} \circ \cdots \circ X^{k}\right.$ : $\left.X^{i} \in \mathcal{C}^{*}(\mathcal{M})\right\}$ of all possible signed covectors, obtained by successively composing signed cocircuits. The set $\mathcal{V}^{*}$ has a natural poset structure, which we denote by $\mathcal{L}\left(\mathcal{V}^{*}\right)$ (in fact, $\mathcal{L}\left(\mathcal{V}^{*}\right)$ is a graded lattice). The order is obtained from the component-wise partial order on vectors in $\{+,-, 0\}^{E}$ with $0<+,-$. We will revisit this poset later in a geometric setting.

The rank of $\mathcal{M}$ is defined to be one less than the length of the longest chain of elements in the poset $\mathcal{L}\left(\mathcal{V}^{*}\right)$. Again, this is not the only way to define the rank of an oriented matroid [4]. We say an element of $E$ is a coloop if it is not present in the support of any signed cocircuit. For brevity, signed cocircuits will also be called cocircuits. It is well known that every matroid has a dual matroid. In the case of oriented matroids, this concept is more delicate, but there is also a notion of duality. One can then talk about circuits, which are the cocircuits of the dual oriented matroid, and the related notions of corank, loops, etc. The corank of an oriented matroid on $n$ elements of rank $r$ is $n-r$.

The cocircuit graph of an oriented matroid $\mathcal{M}$ of rank $r$ is the graph $G^{*}(\mathcal{M})$ whose vertices are the signed cocircuits of $\mathcal{M}$, with an edge connecting signed cocircuits $X$ and $Y$ if $\left|X^{0} \cap Y^{0}\right| \geqslant r-2$ and $S(X, Y)=\emptyset$. An oriented matroid is uniform if $\left|X^{0}\right|=r-1$ for every cocircuit $X \in \mathcal{C}^{*}$. If $X$ and $Y$ are signed cocircuits in $\mathcal{M}$, we use $d_{\mathcal{M}}(X, Y)$ to 
denote the distance from $X$ to $Y$ in $G^{*}(\mathcal{M})$; that is, the length of the shortest path from $X$ to $Y$ in $G^{*}(\mathcal{M})$. We call a path $P$ from $X$ to $Y$ crabbed (introduced in $[23,21]$ ), if for every cocircuit $W \in P, W^{+} \subseteq X^{+} \cup Y^{+}$and $W^{-} \subseteq X^{-} \cup Y^{-}$. The diameter of $G^{*}(\mathcal{M})$ is defined as $\operatorname{diam}\left(G^{*}(\mathcal{M})\right)=\max \left\{d_{\mathcal{M}}(X, Y): X, Y \in \mathcal{C}^{*}(\mathcal{M})\right\}$.

\subsection{Our Results}

This paper presents some new results and also reviews the state of the art of the diameter problem.

(I) To help the reader get started, in Section 2, we quickly review the key notions of oriented matroids that will be relevant for us. Our contributions are as follows:

(II) In Section 3, we present several auxiliary lemmas. One of the first reductions made in studying the Hirsch conjecture was given by Klee and Walkup [20], who showed it was sufficient to study simple polytopes. These are $d$-polytopes in which each vertex is supported by exactly $d$ facets. We make a similar reduction from arbitrary to uniform oriented matroids.

Lemma 4. Let $\mathcal{M}$ be an oriented matroid of rank $r$ on $n$ elements. Then there exists a uniform oriented matroid $\mathcal{M}^{\prime}$ of rank $r$ on $n$ elements such that

$$
\operatorname{diam}\left(G^{*}(\mathcal{M})\right) \leqslant \operatorname{diam}\left(G^{*}\left(\mathcal{M}^{\prime}\right)\right) .
$$

Moreover, when $\mathcal{M}$ is realizable, then $\mathcal{M}^{\prime}$ can be taken to be realizable as well.

Lemma 4, reduces Conjecture 1 to studying uniform oriented matroids. Therefore, for the purposes of studying Conjecture 1, it suffices to consider only uniform oriented matroids.

The following auxiliary lemma shows that the discrepancy between the diameter given in Conjecture 1 and the classical Hirsch bound cannot be improved. Essentially, Conjecture 1 cannot be improved because the distance between antipodal cocircuits is exactly $n-r+2$.

Lemma 5. Let $\mathcal{M}$ be a uniform oriented matroid of rank $r$ on $n$ elements, and let $X, Y \in$ $\mathcal{C}^{*}(\mathcal{M})$. Then

$$
d_{\mathcal{M}}(X, Y) \geqslant \begin{cases}|S(X, Y)|+\left|X^{0} \backslash Y^{0}\right| & \text { if } X \neq-Y, \\ n-r+2 & \text { if } X=-Y .\end{cases}
$$

Moreover, if $\left|X^{0} \backslash Y^{0}\right| \leqslant 1$, then the inequality (1) holds with equality: $d_{\mathcal{M}}(X, Y)=$ $1+|S(X, Y)|$, and in particular, when $X=-Y$, then $d_{\mathcal{M}}(X, Y)=n-r+2$.

(III) Section 4 presents computational results that establish Conjecture 1 for uniform oriented matroids whose ground set has at most nine elements (see Theorem 15). Our proof uses an exhaustive computer search over a database of small oriented matroids.

We then move on to establish Conjecture 1 in low rank. Babson, Finschi, and Fukuda [2, Lemma 6] and Felsner et al. [11, Lemma 4.1] gave proofs of Conjecture 1 for $r \leqslant 3$. 
We present a new geometric proof of that same result (see Theorem 16). We explain why our method does not generalize for rank four matroids. Finally, we also settle the conjecture for oriented matroids of low corank (i.e. $n-r)$. In summary, we have the following theorem:

Theorem 6. Let $\mathcal{M}$ be a uniform oriented matroid of rank $r$ on $n$ elements.

a. If $n \leqslant 9$, then $\operatorname{diam}\left(G^{*}(\mathcal{M})\right)=n-r+2$.

b. If $r \leqslant 3$, then $\operatorname{diam}\left(G^{*}(\mathcal{M})\right)=n-r+2$.

c. If $n-r \leqslant 4$, then $\operatorname{diam}\left(G^{*}(\mathcal{M})\right)=n-r+2$.

(IV) Section 5 discusses a stronger quadratic upper bound on the diameter of a uniform oriented matroid (see Theorem 7). We modify Finschi's proof of Theorem 2 [12, Proposition 2.6.1] to give a slightly stronger bound. Our new bound is tight in rank three, while Finschi's is not. Another improvement is that, as a consequence of Lemma 4, our bound is valid for all oriented matroids rather than just uniform oriented matroids.

Theorem 7. Let $\mathcal{M}$ be an oriented matroid of rank $r$ on $n$ elements, and let $X, Y \in$ $\mathcal{C}^{*}(\mathcal{M})$ with $X \neq-Y$. Then

$$
d_{\mathcal{M}}(X, Y) \leqslant n-r+1+\sum_{k=2}^{\left|X^{0} \backslash Y^{0}\right|-1}\left(\left\lfloor\frac{n-r-k}{2}\right\rfloor+1\right) .
$$

In particular, when $r \geqslant 4$ and $n-r \geqslant 2$,

$$
\operatorname{diam}\left(G^{*}(\mathcal{M})\right) \leqslant n-r+1+\sum_{k=2}^{\min (r-2, n-r)}\left(\left\lfloor\frac{n-r-k}{2}\right\rfloor+1\right) .
$$

This bound contrasts the best-known upper bounds on polytope diameters, which are linear in fixed dimension, but grow exponentially in the dimension (e.g., [18] and [10]). For a survey of the best bounds and more updates about diameters of polytopes see $[7,8,10,26,28]$ and the references therein.

(V) The strong similarities and connections between the diameter problem of convex polytopes and the diameter problem for oriented matroids suggest fascinating questions we try to answer in Section 6:

To start, one may hope that $d_{\mathcal{M}}(X, Y) \leqslant n-r+1$ when $X$ and $Y$ are not antipodal cocircuits. In fact, Finschi posed a similar question in his thesis [12, Open Problem 2], as did Felsner et al. [11, Question 4.2]. Here we answer this question. We show the answer is negative by considering Santos's counterexample to the Hirsch conjecture.

Proposition 8. There is a uniform oriented matroid $\mathcal{M}$ of rank 21 on 40 elements that has a pair of non-antipodal cocircuits $X$ and $Y$ such that $d_{\mathcal{M}}(X, Y) \geqslant 21=n-r+2$. 
It is not immediately clear whether bounds on the diameter of the cocircuit graph of a realizable oriented matroid imply bounds on polytope diameters. This possible connection has been discussed before. For example, a connection of the (original) Hirsch conjecture to Conjecture 1 was stated in Remark 4.3 of [11]. Their proof of their remark shows that, thanks again to Santos's counterexample, there is an example of two cocircuits in the same tope that cannot be connected by a crabbed path of length $n-r+1$. Their remark also suggests two natural strengthenings of Conjecture 1 , both of which would imply the polynomial Hirsch conjecture is true for convex polytopes:

First, if $X$ and $Y$ are vertices in a tope $\mathcal{T}$, does the shortest path from $X$ to $Y$ in the supergraph $G^{*}(\mathcal{M})$ of cocircuits leave the tope $\mathcal{T}$ ? The question is already interesting for a realizable $\mathcal{M}$ where a tope $\mathcal{T}$ corresponds to a polytope. If the shortest path between $X, Y$ always stays in a tope containing both, then a quadratic bound on the diameter of polytopes follows from the quadratic bound for oriented matroids. This would prove the famous polynomial Hirsch Conjecture for those polytopes in the arrangement (recall the polynomial Hirsch conjecture states that the diameter of all convex polytopes is bounded by a polynomial in terms of the number of facets and the dimension, see [26]).

Second, even more strongly, is there always a crabbed path from $X$ to $Y$ whose length is no bigger than the length of any path from $X$ to $Y$ in the entire cocircuit graph $\mathcal{M}$ ? Again, if this was true, the diameter computed over the topes that contain $X, Y$ is always no larger than the diameter of the entire cocircuit graph. Unfortunately, we show the two strengthenings of Conjecture 1 are false. This is the content of Theorem 9. We used a computer search to find the counterexamples and to show they are smallest possible.

Theorem 9. There is a realizable rank 4 uniform oriented matroid $\mathcal{M}$ with 9 elements and a pair its cocircuits $X, Y \in \mathcal{C}^{*}(\mathcal{M})$, whose distance $d_{\mathcal{M}}(X, Y)$ is smaller than the length of any crabbed path from $X$ to $Y$. We prove that no such example with fewer than 9 elements is possible. Moreover, by adding another element to $\mathcal{M}$, we construct a realizable rank 4 oriented matroid $\mathcal{M}^{\prime}$ on 10 elements with two cocircuits $X, Y$ inside a common tope $\mathcal{T}$, such that $d_{\mathcal{M}^{\prime}}(X, Y)<d_{\mathcal{T}}(X, Y)$.

\section{A Quick Review of Oriented Matroids}

Let $E=\left\{\mathbf{v}_{1}, \ldots, \mathbf{v}_{n}\right\} \subseteq \mathbb{R}^{r}$ be any set of vectors. For simplicity, we will assume that $E$ spans $\mathbb{R}^{r}$. We will not make a distinction between $E$ as a set of vectors or $E$ as a matrix in $\mathbb{R}^{r \times n}$. In classical matroid theory, we consider the set of linear dependences among the vectors in $E$. In oriented matroid theory, we consider not only the set of linear dependences on $E$, but also the signs of the coefficients that make up these dependences. To any linear dependence $\sum_{i=1}^{n} z_{i} \mathbf{v}_{i}=\mathbf{0}$ we associate a signed vector $\left(\operatorname{sign}\left(z_{i}\right)\right)_{i=1}^{n}$. The sign of a number $z \in \mathbb{R}$, denoted $\operatorname{sign}(z) \in\{+,-, 0\}$, encodes whether $z$ is positive, negative, or equal to 0 . If $\mathbf{z}=\left(z_{1}, \ldots, z_{n}\right) \in \mathbb{R}^{n}$ is a vector, we use $\operatorname{sign}(\mathbf{z})$ to denote the vector of signs: $\operatorname{sign}(\mathbf{z}):=\left(\operatorname{sign}\left(z_{i}\right)\right)_{i=1}^{n} \in\{+, 0,-\}^{n}$. We define the set of signed vectors on $E$ as

$$
\mathcal{V}(E)=\{\operatorname{sign}(\mathbf{z}): \mathbf{z} \text { is a linear dependence on } E\} .
$$


In other words, $\mathcal{V}(E)=\{\operatorname{sign}(\mathbf{z}): E \mathbf{z}=\mathbf{0}\}$.

Among all signed vectors determined by linear dependences on $E$, those with minimal (and nonempty) support under inclusion, are called the signed circuits of $E$. The set of such signed circuits is denoted $\mathcal{C}(E)$.

Dually, for any $\mathbf{c} \in \mathbb{R}^{r}$, we can consider the signed covector $\left(\operatorname{sign}\left(\mathbf{c}^{T} \mathbf{v}_{i}\right)\right)_{i=1}^{n}$. The set of all signed covectors on $E$ is

$$
\mathcal{V}^{*}(E)=\left\{\operatorname{sign}\left(\mathbf{c}^{T} E\right): \mathbf{c} \in \mathbb{R}^{r}\right\} .
$$

The set of signed covectors of minimal, nonempty support are called signed cocircuits and are denoted by $\mathcal{C}^{*}(E)$. It is important to note that if $X$ is a cocircuit, then so is $-X$.

Summarily, to any collection of vectors $E \subseteq \mathbb{R}^{r}$, there are four sets of vectors that encode dependences among $E$. Those are the signed vectors $\mathcal{V}(E)$ arising from linear dependences, the signed circuits $\mathcal{C}(E)$ arising from minimal linear dependences, the signed covectors $\mathcal{V}^{*}(E)$ arising from valuations of linear functions, and signed cocircuits $\mathcal{C}^{*}(E)$ arising from linear valuations of minimal support. The first fundamental result in oriented matroid theory shows that any one of these sets is sufficient to determine the other three [29, Corollary 6.9]. Any oriented matroid that arises from a collection of signed cocircuits in this way is called a realizable oriented matroid.

Now we are ready to motivate the definition of oriented matroids through a geometric model that proves to be more useful than the axiomatic definition. Let $E=$ $\left\{\mathbf{v}_{1}, \ldots, \mathbf{v}_{n}\right\} \subseteq \mathbb{R}^{r}$ be a collection of vectors, and let $\mathcal{M}(E)$ be the oriented matroid determined by $E$. To each vector $\mathbf{v}_{i}$, there is an associated hyperplane $H_{i}:=\left\{\mathbf{x} \in \mathbb{R}^{r}\right.$ : $\left.\mathbf{x}^{T} \mathbf{v}_{i}=0\right\}$. Each $H_{i}$ is naturally oriented by taking $H_{i}^{+}:=\left\{\mathbf{x} \in \mathbb{R}^{r}: \mathbf{x}^{T} \mathbf{v}_{i}>0\right\}$ and defining $H_{i}^{-}$analogously.

Therefore, the vectors in $E$ determine a central hyperplane arrangement $\mathcal{H}$ in $\mathbb{R}^{r}$. Any vector $\mathbf{x} \in \mathbb{R}^{r}$ has an associated sign vector determined by its position relative to the hyperplanes in $\mathcal{H}$. These signs can be computed as $\operatorname{sign}\left(\mathbf{x}^{T} \mathbf{v}_{i}\right)$ for each $i$; in other words, by computing $\operatorname{sign}\left(\mathbf{x}^{T} E\right)$. Therefore, the signed covectors of $\mathcal{M}(E)$ are in bijection with the regions of the hyperplane arrangement $\mathcal{H}$.

Further, because sign $\left(\mathbf{x}^{T} E\right)=\operatorname{sign}\left((c \mathbf{x})^{T} E\right)$ for any positive scalar $c$, no information from $\mathcal{H}$ is lost if we intersect $\mathcal{H}$ with the unit sphere $\mathbb{S}^{r-1}$, giving a collection of codimension-one spheres $\left\{s_{i}=H_{i} \cap \mathbb{S}^{r-1}: H_{i} \in \mathcal{H}\right\}$. This induces a cell decomposition of $\mathbb{S}^{r-1}$ whose nonempty faces correspond to covectors of $\mathcal{M}(E)$ and whose vertices correspond to cocircuits of $\mathcal{M}(E)$. The regions corresponding to covectors of maximal support are called topes. An example is illustrated in Figure 2. In that figure, the cocircuit $X$ is encoded by the sign vector $(+,+, 0,-, 0)$. Similarly, the shaded region (a tope) corresponds to the covector $(+,+,+,-,+)$.

Not all matroids can be oriented. Determining whether a matroid is orientable is an NP-complete problem, even for fixed rank (see [24]). But, a topological model provides the "right" intuition for visualizing arbitrary oriented matroids. Every oriented matroid can be viewed as an arrangement of equators on a sphere, as in the realizable case, provided that one is allowed to slightly perturb the spheres determined by $H_{i} \cap \mathbb{S}^{r-1}$ in the following way. 


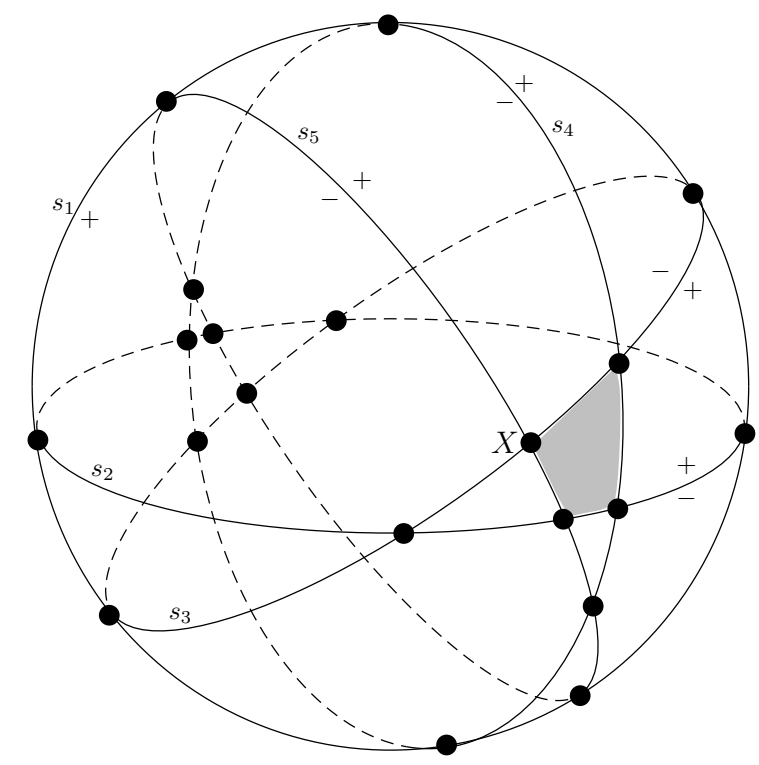

Figure 2: An oriented matroid arising from an arrangement of five hyperplanes.

Let $Q$ be an equator of $\mathbb{S}^{r-1}$; that is, the intersection of $\mathbb{S}^{r-1}$ with some $(r-1)$ dimensional subspace of $\mathbb{R}^{r}$. If $\varphi: \mathbb{S}^{r-1} \rightarrow \mathbb{S}^{r-1}$ is a homeomorphism, then the image of the equator $\varphi(Q) \subseteq \mathbb{S}^{r-1}$ is called a pseudosphere. Because $Q$ decomposes $\mathbb{S}^{r-1}$ into two pieces, so too does $\varphi(Q)$. Therefore, we may define an oriented pseudosphere to be a pseudosphere, $s$, together with a choice of a positive side $s^{+}$and negative side $s^{-}$. Now we may define an arrangement of pseudospheres in $\mathbb{S}^{r-1}$ to be a finite collection of pseudospheres $\mathcal{P}=\left\{s_{e}: e \in E\right\} \subseteq \mathbb{S}^{r-1}$ such that

1. for any subset $A \subseteq E$, the set $S_{A}=\bigcap_{e \in A} s_{e}$ is a topological sphere, and

2. if $S_{A} \nsubseteq s_{e}$ for $A \subseteq E$ and $e \in E$, then $S_{A} \cap s_{e}$ is a pseudosphere in $S_{A}$ with two parts, $S_{A} \cap s_{e}^{+}$and $S_{A} \cap s_{e}^{-}$.

A pseudosphere arrangement is essential if $\bigcap_{e \in E} s_{e}=\emptyset$. Any essential pseudosphere arrangement $\mathcal{P}$ induces a regular cell decomposition on $\mathbb{S}^{r-1}$. Because each pseudosphere in $\mathcal{P}$ has a positive and negative side, the cells of this decomposition are naturally indexed by sign vectors in $\{+,-, 0\}^{E}$. We use $\Gamma(\mathcal{P})$ to denote the poset of such sign vectors, ordered by face containment. We have encountered this same (abstract) poset before as $\mathcal{L}\left(\mathcal{V}^{*}\right)$ in the introduction, the poset induced over the set of covectors $\mathcal{V}^{*}$ of an oriented matroid. As it turns out the following theorem of Folkman and Lawrence gives an exact correspondence between oriented matroids and pseudosphere arrangements. The same sets of sign vectors appear in both cases.

Theorem 10. (Topological Representation Theorem [15])

Let $\mathcal{P}$ be an essential arrangement of pseudospheres in $\mathbb{S}^{r-1}$. Then $\Gamma(\mathcal{P}) \cup\{\mathbf{0}\}$ is the set of covectors of an oriented matroid of rank $r$. Conversely, if $\mathcal{V}^{*}$ is the set of covectors 
of a loopless oriented matroid of rank $r$, then there exists an essential arrangement of pseudospheres $\mathcal{P}$ on $\mathbb{S}^{r-1}$ with $\Gamma(\mathcal{P})=\mathcal{V}^{*} \backslash\{\mathbf{0}\}$.

If $\mathcal{M}$ is an oriented matroid, the pseudosphere arrangement $\mathcal{P}$ guaranteed by the Topological Representation Theorem is called the Folkman-Lawrence representation of $\mathcal{M}$. Two elements $e, f \in E$ are parallel if $X_{e}=X_{f}$ for all $X \in \mathcal{V}^{*}$ or $X_{e}=-X_{f}$ for all $X \in \mathcal{V}^{*}$. Note that we can eliminate parallel elements without changing the pseudosphere arrangement $\mathcal{P}$.

Remark 11. Let $\mathcal{M}$ be a uniform oriented matroid of rank $r$. If $A \subseteq E(\mathcal{M})$ is any set with $|A| \leqslant r-1$, then $S_{A}=\bigcap_{e \in A} s_{e}$ is an $(r-1-|A|)$-dimensional pseudosphere in the Folkman-Lawrence representation $\mathcal{P}(\mathcal{M})$.

Let $\mathcal{M}$ be an oriented matroid of rank $r$, and let $\mathcal{P}$ be the Folkman-Lawrence representation of $\mathcal{M}$. Then the underlying graph of $\mathcal{P}$ (as a cell complex) is the cocircuit graph $G^{*}(\mathcal{M})$. This provides a geometric model for visualizing cocircuit graphs of oriented matroids. A coline in $\mathcal{M}$ is a one-dimensional sphere in the Folkman-Lawrence representation of $\mathcal{M}$. In matroidal language, a coline is a covector that covers a cocircuit in the natural component-wise partial order where $0<+,-$. For a uniform oriented matroid of rank $r$, a coline is a covector $U$ with $\left|U^{0}\right|=r-2$. Further, in a uniform oriented matroid, for each subset $S \in\left(\begin{array}{c}{[n]} \\ r-2\end{array}\right)$, there exists a coline $U$ with $U^{0}=S$. The graph of any coline is a simple cycle of length $2(n-r+1)$.

The Folkman-Lawrence representation gives us a more concrete topological understanding of the following operations on oriented matroids. Let $\mathcal{M}$ be an oriented matroid on ground set $E$ with signed covectors $\mathcal{V}^{*}(\mathcal{M})$, and let $A \subseteq E$. The restriction of a sign vector $X \in\{+,-, 0\}^{E}$ to $A$ is the sign vector $\left.X\right|_{A} \in\{+,-, 0\}^{A}$ defined by $\left(\left.X\right|_{A}\right)_{e}=X_{e}$ for all $e \in A$. The deletion $\mathcal{M} \backslash A$ is the oriented matroid with covectors

$$
\mathcal{V}^{*}(\mathcal{M} \backslash A)=\left\{\left.X\right|_{E \backslash A}: X \in \mathcal{V}^{*}(\mathcal{M})\right\} \subseteq\{+,-, 0\}^{E \backslash A} .
$$

The contraction $\mathcal{M} / A$ is the oriented matroid with covectors

$$
\mathcal{V}^{*}(\mathcal{M} / A)=\left\{\left.X\right|_{E \backslash A}: X \in \mathcal{V}^{*}(\mathcal{M}), A \subseteq X^{0}\right\} \subseteq\{+,-, 0\}^{E \backslash A} .
$$

The fact that $\mathcal{M} \backslash A$ and $\mathcal{M} / A$ are oriented matroids is proved in [4, Lemma 4.1.8].

The deletion $\mathcal{M} \backslash A$ is also referred to as the restriction of $\mathcal{M}$ to $E \backslash A$. Geometrically, $\mathcal{M} \backslash A$ is the oriented matroid of the same rank as $\mathcal{M}$ obtained by removing pseudospheres $\left\{s_{e}: e \in A\right\}$. The contraction $\mathcal{M} / A$ is the oriented matroid obtained by intersecting $S_{A}$ with $\left\{s_{e}: e \in E \backslash A\right\}$.

Note also that the pseudosphere arrangement of an oriented matroid of rank $r$ lies on the sphere $\mathbb{S}^{r-1}$. The topes correspond to the regions, homeomorphic to balls of dimension $r-1$, that partition the sphere. For realizable oriented matroids coming from a hyperplane arrangement, topes are actual convex polytopes.

Given a tope $\mathcal{T}$ of an oriented matroid $\mathcal{M}$, we define its graph as the subgraph of $G^{*}(\mathcal{M})$ induced by the cociruits of $\mathcal{M}$ in $\mathcal{T}$. Next, we show the graph of a tope $\mathcal{T}$ in a uniform oriented matroid $\mathcal{M}$ of rank $r$ on $n$ elements, is isomorphic to a graph of an 
abstract polytope of dimension $r-1$ on $n$ elements. Abstract polytopes, an abstraction of simple polytopes, were introduced by Adler and Dantzig [1] for the purpose of studying the diameter of their graphs. Abstract polytopes have been further generalized in recent years by several authors (see $[10,26]$ and references there for details).

Definition 12. Let $T$ be a finite set. A family $\mathcal{A}$ of subsets of $T$ (called vertices) forms a $d$-dimensional abstract polytope on the ground set $T$ if the following three axioms are satisfied:

(i) Every vertex of $\mathcal{A}$ has cardinality $d$.

(ii) Any subset of $d-1$ elements of $T$ is either contained in no vertices of $\mathcal{A}$ or in exactly two (called neighbors or adjacent vertices).

(iii) Given any pair of distinct vertices $X, Y \in \mathcal{A}$, there exists a sequence of vertices $X=Z_{0}, Z_{1}, \ldots, Z_{k}=Y$ in $\mathcal{A}$ such that

(a) $Z_{i}, Z_{i+1}$ are adjacent for all $i=0,1, \ldots, k-1$, and

(b) $X \cap Y \subset Z_{i}$ for all $i=0,1, \ldots, k$.

The graph $G_{a b s}(\mathcal{A})$ of an abstract polytope $\mathcal{A}$ is composed of nodes corresponding to its vertices, where two vertices are adjacent on the graph as specified in axiom (ii).

Consider a simple polytope $\mathcal{P}$ of dimension $d$ which is the intersection of $n$ facetdefining half-spaces. Then, indexing the $n$ facets by $1, \ldots, n$, the family of all sets of indices that define a vertex of $\mathcal{P}$ is an abstract polytope of dimension $d$ on the ground set $\{1, \ldots, n\}$. In particular, the three axioms of abstract polytopes state that the graph $G(\mathcal{P})$ associated with the vertices of $\mathcal{P}$ has the following three properties:

(i) $G(\mathcal{P})$ is regular of degree $d$ (as all the hyperplanes corresponding to the half-spaces are in general position.)

(ii) All edges of $G(\mathcal{P})$ have two vertices as end points (as $\mathcal{P}$ is bounded).

(iii) For any two vertices $X, Y$ that lie in a face $F$ of $\mathcal{P}$, there exists a path between the nodes corresponding to $X$ and $Y$ on $G(\mathcal{P})$ composed entirely of nodes corresponding to vertices on $F$ (as $F$ is also a polytope.)

Interestingly, while the axioms of abstract polytopes represent only three basic properties related to graphs of simple polytopes, a substantial number of the results related to diameter of simple polytopes in [20] have been proved in [1] for abstract polytopes.

Next, we show that these properties are satisfied by the graph of topes of uniform oriented matroids. The graph of a tope, its connectivity, and the relation to pseudomanifolds has been studied in [5]. 
Lemma 13. Given a uniform oriented matroid $\mathcal{M}=\left(E, \mathcal{C}^{*}\right)$ of rank $r \geqslant 2$ and a tope $\mathcal{T}$ of $\mathcal{M}$, let

$$
\mathcal{C}_{\mathcal{T}}=\left\{X \in \mathcal{C}^{*}: X<\mathcal{T}\right\}, \text { and } \mathcal{A}=\left\{X^{0}: X \in \mathcal{C}_{\mathcal{T}}\right\} .
$$

Then, $\mathcal{A}$ is a d-dimensional abstract polytope on the ground set $E$, where $d=r-1$. Moreover, the graph $G(\mathcal{T})$ of $\mathcal{T}$ is isomorphic to the graph $G_{a b s}(\mathcal{A})$ of $\mathcal{A}$.

Proof. We show that $\mathcal{A}$ satisfies the three axioms of abstract polytopes:

(i) Axiom (i) holds because $\mathcal{M}$ is a uniform oriented matroid of rank $r$.

(ii) Let $E^{\prime} \subset E$ such that $\left|E^{\prime}\right|=d-1$, and assume that there exists $X \in \mathcal{C}_{\mathcal{T}}$ such that $E^{\prime} \subset X^{0}$ (otherwise, no vertex of $\mathcal{A}$ contains $E^{\prime}$ and we are done). Let $U=\{W \in$ $\left.\mathcal{M}^{*}: E^{\prime} \subset W^{0}\right\}$, then $U$ is a coline of $\mathcal{M}$ whose graph is a simple cycle. Let $Y_{1}, Y_{2}$ be the two adjacent cocircuits to $X$ in $U$. Then, there exists an element $e \in E \backslash E^{\prime}$ such that $S\left(Y_{1}, Y_{2}\right)=e$ and $S\left(X, Y_{i}\right)=\emptyset(i=1,2)$, implying that exactly one of $Y_{1}, Y_{2}$, say $Y_{1}$, is in $\mathcal{T}$. However, no other cocircuit in $U$ is in $\mathcal{T}$. Suppose, to the contrary, that there exists $Z \in U$, distinct from $X$ and $Y_{1}$ that belongs to $\mathcal{T}$. Then by definition

$$
\left|X^{0} \cap Y_{1}^{0}\right|=\left|X^{0} \cap Z^{0}\right|=\left|Y_{1}^{0} \cap Z^{0}\right|=d-1 \text {, and } S\left(X, Y_{1}\right)=S(X, Z)=S\left(Y_{1}, Z\right)=\emptyset \text {. }
$$

This means that $X, Y_{1}$, and $Z$, are all adjacent on $U$. As the graph of $U$ is a simple cycle of size $2(n-r+1)$, this leads to contradiction.

(iii) By [11, Theorem 2.3], for any $X, Y \in \mathcal{C}^{*}$ there exists an $(X, Y)$ crabbed path on $G^{*}(\mathcal{M})$. That is, there exists a path $X=Z_{0}, Z_{1}, \ldots, Z_{k}=Y$ on $G^{*}(\mathcal{M})$ such that $Z_{i}^{+} \subseteq X^{+} \cup Y^{+}$and $Z_{i}^{-} \subseteq X^{-} \cup Y^{-}$for all $0 \leqslant i \leqslant k$. This implies that if $X, Y \in \mathcal{C}_{\mathcal{T}}$, then for $i=1, \ldots, k-1, Z_{i} \in \mathcal{C}_{\mathcal{T}}\left(\right.$ as $Z_{i}<\mathcal{T}$ ), so $Z_{i}^{0} \in \mathcal{A}$, and $X^{0} \cap Y^{0} \subseteq Z_{i}^{0}$. Now, let $G(\mathcal{T})$ be the graph of $\mathcal{T}$. Note that as $S(X, Y)=\emptyset$ for any $X, Y \in \mathcal{C}_{\mathcal{T}}, X$ and $Y$ share an edge on $G(\mathcal{T})$ if and only if $\left|X^{0} \cap Y^{0}\right|=d-1$. However, the two vertices on $G_{a b s}(\mathcal{A})$ corresponding to $X^{0}, Y^{0}$ are adjacent if and only if $\left|X^{0} \cap Y^{0}\right|=d-1$. Thus, we conclude that $G(\mathcal{T})$ is isomorphic to $G_{a b s}(\mathcal{A})$, so Axiom (iii) is satisfied.

Note that by the proof of part (iii) above we have that the graph $G(\mathcal{T})$ of $\mathcal{T}$ is isomorphic to the graph $G_{a b s}(\mathcal{A})$ of $\mathcal{A}$.

\section{Reductions and Lower Bounds}

For the ease of notation, let $O M(n, r)$ be the set of all oriented matroids of rank $r$ whose ground set has cardinality $n$. Let $\operatorname{UOM}(n, r)$ be the set of all uniform oriented matroids in $O M(n, r)$. Let $\Delta(n, r)$ denote the maximal diameter of $G^{*}(\mathcal{M})$ as $\mathcal{M}$ ranges over $O M(n, r)$. Klee and Walkup [20] showed that the maximal diameter among all $d$ dimensional polytopes with $n$ facets is achieved by a simple polytope. Their argument was straightforward: if $P$ is a $d$-polytope with $n$ facets that is not simple, then slightly perturbing the facets of $P$ will produce a simple polytope whose diameter is at least as 
large as that of $P$. Our goal in this section is to prove an analogous result for oriented matroids. First we require some definitions, see [4, Section 7.1 and 7.2] for more details.

Let $\mathcal{M}$ be an oriented matroid on ground set $E$. An extension of $\mathcal{M}$ is an oriented matroid $\widetilde{\mathcal{M}}$ on a ground set $\widetilde{E}$ that contains $E$, such that the restriction of $\widetilde{\mathcal{M}}$ to $E$ is $\mathcal{M}$. We say $\widetilde{\mathcal{M}}$ is a single element extension if $|\widetilde{E} \backslash E|=1$. For any single element extension $\widetilde{\mathcal{M}}$, there is a unique way to extend cocircuits of $\mathcal{M}$ to cocircuits of $\widetilde{\mathcal{M}}$. Specifically, there is a function

$$
\sigma: \mathcal{C}^{*}(\mathcal{M}) \rightarrow\{+,-, 0\}
$$

such that $\sigma(-Y)=-\sigma(Y)$ for all $Y \in \mathcal{C}^{*}(\mathcal{M})$ and

$$
\left\{(Y, \sigma(Y)): Y \in \mathcal{C}^{*}(\mathcal{M})\right\} \subseteq \mathcal{C}^{*}(\widetilde{\mathcal{M}})
$$

That is, $(Y, \sigma(Y))$ is a cocircuit of $\widetilde{\mathcal{M}}$ for every cocircuit $Y$ of $\mathcal{M}$. The functions $\sigma$ : $\mathcal{C}^{*} \rightarrow\{+,-, 0\}$ that correspond to single element extensions are called localizations. Furthermore, $\widetilde{\mathcal{M}}$ is uniquely determined by $\sigma$, with

$$
\begin{aligned}
& \mathcal{C}^{*}(\widetilde{\mathcal{M}})=\left\{(Y, \sigma(Y)): Y \in \mathcal{C}^{*}(\mathcal{M})\right\} \cup \\
& \left\{\left(Y^{1} \circ Y^{2}, 0\right): Y^{1}, Y^{2} \in \mathcal{C}^{*}(\mathcal{M}), \sigma\left(Y^{1}\right)=-\sigma\left(Y^{2}\right) \neq 0, S\left(Y^{1}, Y^{2}\right)=\emptyset, \rho\left(Y^{1} \circ Y^{2}\right)=2\right\} .
\end{aligned}
$$

Here $\rho$ is the rank function and $\circ$ is the composition of covectors.

Now we are ready to define the perturbation map on non-uniform oriented matroids.

Definition 14. [4, Theorem 7.3.1] Let $\mathcal{M}$ be an oriented matroid of rank $r \geqslant 2$ on $E$. If $f \in E$ is not a coloop, then $\mathcal{M}$ is a single element extension of a rank $r$ oriented matroid $\mathcal{M}_{0}:=\mathcal{M} \backslash f$, with localization $\sigma_{f}$. Let $\bar{W} \in \mathcal{C}^{*}\left(\mathcal{M}_{0}\right)$ be a cocircuit with $\sigma_{f}(\bar{W})=0$, meaning $W=(\bar{W}, 0)$ is a cocircuit of $\mathcal{M}$. Then the local perturbation $\mathcal{M}^{\prime}$ of $\mathcal{M}$ can be defined as a single element extension of $\mathcal{M}_{0}$ with localization

$$
\sigma_{L P}(\bar{Y})= \begin{cases}+ & \text { if } \bar{Y}=\bar{W}, \\ - & \text { if } \bar{Y}=-\bar{W}, \\ \sigma_{f}(\bar{Y}) & \text { otherwise. }\end{cases}
$$

We can now reduce the general diameter problem to the case of uniform oriented matroids, as promised by Lemma 4 .

Proof. (of Lemma 4)

Let $\mathcal{M}$ be a non-uniform oriented matroid. We may assume without loss of generality that, $\mathcal{M}$ does not have any loops, coloops or parallel elements since removing them will not affect the cocircuit graph of $\mathcal{M}$. Note that there exists $W \in \mathcal{C}^{*}(\mathcal{M})$ with $\left|W^{0}\right|>r-1$. Pick an arbitrary $f \in W^{0}$. Let $\mathcal{M}_{0}:=\mathcal{M} \backslash f$ and let $\mathcal{M}^{\prime}$ be the perturbed oriented matroid defined in Definition 14. We will show $\operatorname{diam}(\mathcal{M}) \leqslant \operatorname{diam}\left(\mathcal{M}^{\prime}\right)$. In addition, if $\mathcal{M}$ is realizable, then we will show the perturbed $\mathcal{M}^{\prime}$ can also be made realizable. From this, it will follow that for all $n$ and $r$, the optimal bound $\Delta(n, r)$ is achieved by a uniform oriented matroid. 

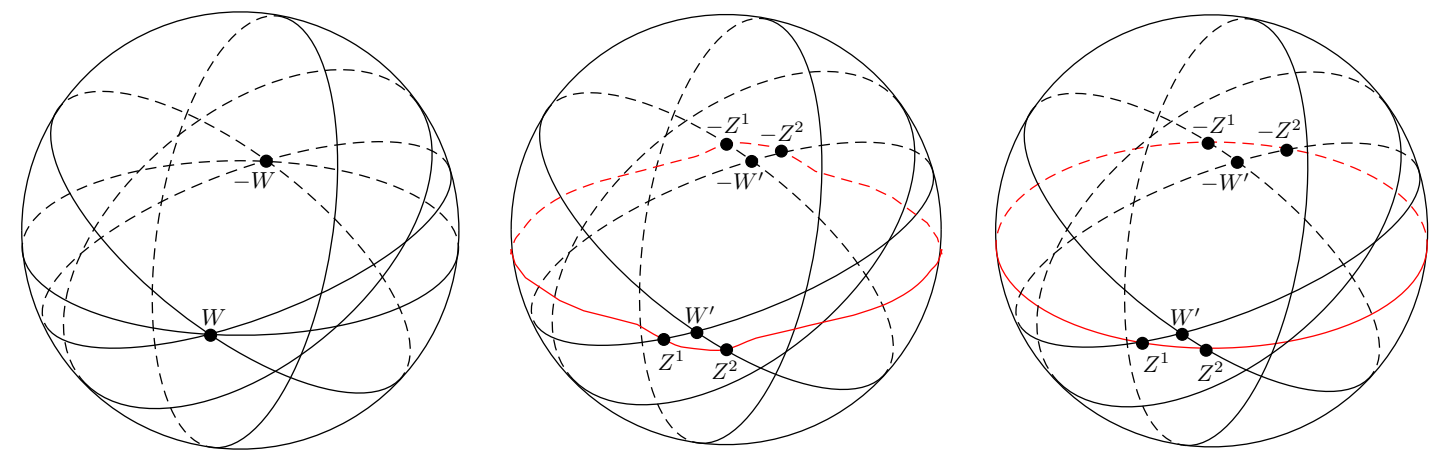

Figure 3: A non-uniform oriented matroid (left), a local perturbation (center), and a realizable local perturbation (right).

Denote by $\left\{X^{1}, X^{2}, \ldots, X^{k}\right\}=\left\{X \in \mathcal{C}^{*}\left(\mathcal{M}_{0}\right): \sigma_{f}(X)=-, S(\bar{W}, X)=\emptyset, \rho(\bar{W}, X)=\right.$ $2\}$. Note that $X^{1}, \ldots, X^{k}$ are exactly the cocircuits that are adjacent to $\bar{W}$ in $G^{*}\left(\mathcal{M}_{0}\right)$ before the extension with $\sigma_{f}\left(X^{i}\right)=-$. Let $Z^{i}=\left(X^{i} \circ \bar{W}, 0\right)$. After the perturbation by $\sigma_{L P}, W$ is mapped to $W^{\prime}=(\bar{W},+)$. Since $\sigma_{L P}$ and $\sigma_{f}$ only differ on $\pm \bar{W}$, it follows that $\pm Z^{1}, \ldots, \pm Z^{k}$ are all the cocircuits created by this perturbation. After the perturbation, each edge of the form $\left\{W, X^{i}\right\}$ in $G^{*}(\mathcal{M})$ is subdivided into two edges $\left\{W, Z^{i}\right\}$ and $\left\{Z^{i}, X^{i}\right\}$ (similarly $\left\{-W,-X^{i}\right\}$ is subdivided into $\left\{-W^{\prime}, Z^{i}\right\}$ and $\left\{-Z^{i},-X^{i}\right\}$ ).

Now let $X, Y \in \mathcal{C}^{*}(\mathcal{M})$ be any two cocircuits of $\mathcal{M}$ such that $X, Y \in \mathcal{C}^{*}\left(\mathcal{M}^{\prime}\right)(X, Y$ could be $\pm W$, in this case we just consider $\pm W^{\prime}$ in $\left.\mathcal{M}^{\prime}\right)$. Take a minimal path between $X$ and $Y$ on $G^{*}\left(\mathcal{M}^{\prime}\right)$, and replace any elements of $\left\{ \pm W^{\prime}, \pm Z^{1}, \ldots, \pm Z^{k}\right\}$ with $\pm W$ respectively. This gives us a path (potentially having repeated elements and not necessarily shortest) between $X$ and $Y$ in $\mathcal{M}$. Now if we pick $X, Y \in \mathcal{C}^{*}(\mathcal{M})$ that realize the diameter of $\mathcal{M}$, since $d_{\mathcal{M}}(X, Y) \leqslant d_{\mathcal{M}^{\prime}}(X, Y)$, we have $\operatorname{diam}(\mathcal{M})=d_{\mathcal{M}}(X, Y) \leqslant d_{\mathcal{M}^{\prime}}(X, Y) \leqslant$ $\operatorname{diam}\left(\mathcal{M}^{\prime}\right)$.

Now suppose $\mathcal{M}$ is realizable. Let $\mathcal{H}=\left\{H_{1}, \ldots, H_{n}\right\}$ be the hyperplane arrangement corresponding to $\mathcal{M}$ (with $f$ corresponding to $H_{n}$ ). Let $H_{i}=\left\{\mathbf{x}: \mathbf{x}^{T} \mathbf{v}_{i}=0\right\}$, and $\mathbf{w}$ be the vector realizing $W$. Note that we have $\mathbf{w}^{T} \mathbf{v}_{n}=0$ since the last entry of $W$ is 0 . Consider $\mathbf{y}$, the minimizer of $\mathbf{x}^{T} \mathbf{v}_{n}$ over all cocircuits of $\mathcal{M}$ subject to $\mathbf{x}^{T} \mathbf{v}_{n}>0$. Now we replace $H_{n}$ by $H_{n}^{\prime}=\left\{\mathbf{x}: \mathbf{x}^{T}\left((1-\epsilon) \mathbf{v}_{n}+\epsilon \mathbf{y}\right)=0\right\}$, in which the choice of $\epsilon$ will be made later. Note that,

$$
\mathbf{x}^{T}\left((1-\epsilon) \mathbf{v}_{n}+\epsilon \mathbf{y}\right)=\mathbf{x}^{T} \mathbf{v}_{n}-\epsilon \mathbf{x}^{T} \mathbf{v}_{n}+\epsilon \mathbf{x}^{T} \mathbf{y}
$$

We first pick the sign of $\epsilon$ so that $\epsilon \mathbf{w}^{T} \mathbf{y}>0$; as a result, $\mathbf{w} \in H_{n}^{\prime+}$ and $-\mathbf{w} \in H_{n}^{\prime-}$. Then we take $|\epsilon|$ small enough such that $\left|\mathbf{x}^{T} \mathbf{v}_{n}\right|>\left|\epsilon\left(\mathbf{x}^{T} \mathbf{v}_{n}-\mathbf{x}^{T} \mathbf{w}^{\prime}\right)\right|$ for all $\mathbf{x}$ vectors that realize a cocircuit in $\mathcal{M}$ (this choice of $\epsilon$ exists since the number of cocircuits is finite and we may scale the vector). The construction ensures that all cocircuits, except those that lie on $H_{n}$ with degeneracy, will have the same sign as defined in Definition 14. As a result $\mathcal{H}^{\prime}=\left\{H_{1}, \ldots, H_{n-1}, H_{n}^{\prime}\right\}$ corresponds to some realizable oriented matroid $\mathcal{M}^{\prime}$ after some local perturbations (the composition of perturbation maps on all cocircuits with degeneracy on $H_{n}$ (including $W$ ) as defined in Definition 14). 
To conclude, we have decreased the number of pairs of $(W, f)$ with $\left|W^{0}\right|>r-1$ and $W_{f}=0$ without decreasing the diameter. By continuing this procedure, we will eventually obtain an oriented matroid in which no such pair of $(W, f)$ can be found, or equivalently $\left|X^{0}\right|=r-1$ for all $X \in \mathcal{C}^{*}(\mathcal{M})$. Hence $\Delta(n, r)$ will be achieved by a uniform oriented matroid.

Hence it suffices to study uniform oriented matroids for the purpose of bounding $\Delta(n, r)$. The bound in Conjecture 1 can be rewritten as $\Delta(n, r) \leqslant n-(r-1)+1$. For polytopes, $n-(r-1)+1=n-d+1$. It may seem mysterious that the bound here is one more than the Hirsch bound, so we will pause for a moment to discuss this. We begin by proving Lemma 5 from the Introduction.

\section{Proof. (of Lemma 5)}

Recall that if cocircuits $Z$ and $W$ are adjacent in $G^{*}(\mathcal{M})$, then there are elements $e \in Z^{0} \backslash W^{0}$ and $e^{\prime} \in W^{0} \backslash Z^{0}$ such that $Z^{0}=\left(W^{0} \backslash\left\{e^{\prime}\right\}\right) \cup\{e\}$. In other words, when we move from $Z$ to $W$, we see $Z_{e}=0$ change to become $W_{e} \neq 0$ and $Z_{e^{\prime}} \neq 0$ change to become $W_{e^{\prime}}=0$. Therefore, we will say that each edge in $G^{*}(\mathcal{M})$ encodes two "basic transformations", which are changes to the cocircuit that transform a nonzero entry into a zero entry or vice versa.

Now we consider the differences in the sign patterns of $X$ and $Y$. For each $e \in S(X, Y)$ we require two basic transformations to move from $X$ to $Y$ : one to transform $X_{e}$ to 0 , and another to transform 0 to $-X_{e}=Y_{e}$. For each $e \in X^{0} \backslash Y^{0}$, we require one basic transformation to transform 0 to $Y_{e}$. Similarly, for each $e \in Y^{0} \backslash X^{0}$, we require one basic transformation to transform $X_{e}$ to 0 . Therefore, moving from $X$ to $Y$ requires at least $2|S(X, Y)|+\left|X^{0} \backslash Y^{0}\right|+\left|Y^{0} \backslash X^{0}\right|=2|S(X, Y)|+2\left|X^{0} \backslash Y^{0}\right|$ basic transformations. Thus $d_{\mathcal{M}}(X, Y) \geqslant|S(X, Y)|+\left|X^{0} \backslash Y^{0}\right|$.

Now we examine the case where $X=-Y$ more closely. In this case, $S(X, Y)=$ $\operatorname{supp}(X)$ and $X^{0}=Y^{0}$. Pick a shortest path from $X$ to $Y$ in $G^{*}(\mathcal{M})$ and let $Z$ be the neighbor of $X$ on this path. Then $|S(Y, Z)|=n-r$ and $\left|Z^{0} \backslash Y^{0}\right|=1$, so $d_{\mathcal{M}}(Y, Z) \geqslant$ $n-r+1$ by the above argument. Therefore, $d_{\mathcal{M}}(X, Y)=1+d_{\mathcal{M}}(Y, Z) \geqslant n-r+2$.

Next, consider the case $\left|X^{0} \backslash Y^{0}\right| \leqslant 1$. We show that the equality holds for expression (1).

Let $A \subseteq X^{0} \cap Y^{0}$ have cardinality $r-2$. If $\left|X^{0} \backslash Y^{0}\right|=1$, then $A=X^{0} \cap Y^{0}$; otherwise, $X=-Y$ and we can pick $r-2$ elements arbitrarily from $X^{0}=Y^{0}$. Let $\left\{s_{e}: e \in E\right\}$ be the pseudospheres in the Folkman-Lawrence representation of $\mathcal{M}$ and let $S_{A}=\bigcap_{e \in A} s_{e}$. Because $\mathcal{M}$ is uniform, we know $S_{A} \approx \mathbb{S}^{1}$.

We saw above that in general $d_{\mathcal{M}}(X, Y) \geqslant 1+|S(X, Y)|$. On the other hand, the elements of $S(X, Y)$ are in bijective correspondence with cocircuits along the shortest path from $X$ to $Y$ in $S_{A}$. Indeed, if $Z$ is such a cocircuit, then $Z$ and $-Z$ are antipodal vertices on $S_{A}$, so they constitute a 0 -dimensional pseudosphere whose positive side contains one of $X$ or $Y$ and whose negative side contains the other. Thus the distance from $X$ to $Y$ on $S_{A}$ is exactly $1+|S(X, Y)|$. This proves $d_{\mathcal{M}}(X, Y) \leqslant 1+|S(X, Y)|$. 


\section{Results for small matroids}

\subsection{Computer-based results for oriented matroids with few elements}

Finschi and Fukuda [13] computed the exact number of isomorphism classes of uniform oriented matroids, and gave a representative of each isomorphism class, when $n \leqslant 9$ and in small rank/corank when $n=10$. We established Conjecture 1 for all of these examples using computers.

\begin{tabular}{|c|c|c|c|c|c|c|c|c|c|}
\hline & $n=2$ & $n=3$ & $n=4$ & $n=5$ & $n=6$ & $n=7$ & $n=8$ & $n=9$ & $n=10$ \\
\hline$r=2$ & 1 & 1 & 1 & 1 & 1 & 1 & 1 & 1 & 1 \\
\hline$r=3$ & & 1 & 1 & 1 & 4 & 11 & 135 & 4382 & 312356 \\
\hline$r=4$ & & & 1 & 1 & 1 & 11 & 2628 & 9276595 & unknown \\
\hline$r=5$ & & & & 1 & 1 & 1 & 135 & 9276595 & unknown \\
\hline$r=6$ & & & & & 1 & 1 & 1 & 4382 & unknown \\
\hline$r=7$ & & & & & & 1 & 1 & 1 & 312356 \\
\hline$r=8$ & & & & & & & 1 & 1 & 1 \\
\hline$r=9$ & & & & & & & & 1 & 1 \\
\hline$r=10$ & & & & & & & & & 1 \\
\hline
\end{tabular}

Table 1: Number of uniform oriented matroids for $n \leqslant 10$.

Each isomorphism class is encoded by its chirotope representation. Chirotopes, or basis orientations, are one of the equivalent axiomatic systems for oriented matroids (see [4, Section 3] for more details). For a given oriented matroid on ground set $E$, the chirotope defines a mapping $\chi: E^{r} \rightarrow\{-, 0,+\}$. For a realizable oriented matroid with vector configuration $\left\{\mathbf{v}_{1}, \ldots, \mathbf{v}_{n}\right\}$,

$$
\chi\left(\lambda_{1}, \ldots, \lambda_{r}\right)=\operatorname{sign}\left(\operatorname{det}\left(\mathbf{v}_{\lambda_{1}}, \mathbf{v}_{\lambda_{2}}, \ldots, \mathbf{v}_{\lambda_{r}}\right)\right) .
$$

The data can be found on Finschi and Fukuda's Homepage of Oriented Matroids [14]. Given a chirotope map $\chi$ of an oriented matroid of rank $r$ on $E=\{1,2, \ldots, n\}$, we can generate the cocircuits by computing the set $\mathcal{C}^{*}(\chi)=\{(\chi(\lambda, 1), \chi(\lambda, 2), \ldots, \chi(\lambda, n))$ : $\left.\lambda \in E^{r-1}\right\}$. Since $\mathcal{M}$ is uniform, we add an edge between $X, Y \in \mathcal{C}^{*}(\mathcal{M})$ if and only if $\left|X^{0} \cap Y^{0}\right|=r-2$ and $|S(X, Y)|=0$. For $n=9, r=5$ and $n=10, r=7$, the chirotope maps are missing in the original dataset. However we can look at their duals $(n=9$, $r=4$ and $n=10, r=3$ ) and consider the set of circuits instead. The pseudocode for computing the set of cocircuits and circuits is given below in Algorithms 1 and 2.

After finding all the cocircuits and edges, we used the Python NetworkX package [9] to construct the cocircuit graph. This package has a method for computing the diameter of a graph, and also for determining the distance between any pairs of vertices. Table 1 shows the number of isomorphism classes (up to reorientation) of uniform oriented matroids of cardinality $n$ and rank $r$. We used a MacBook Pro with quad-core $2.2 \mathrm{GHz}$ Intel i7 processor, as well as UC Davis Math servers to construct the cocircuit graphs 


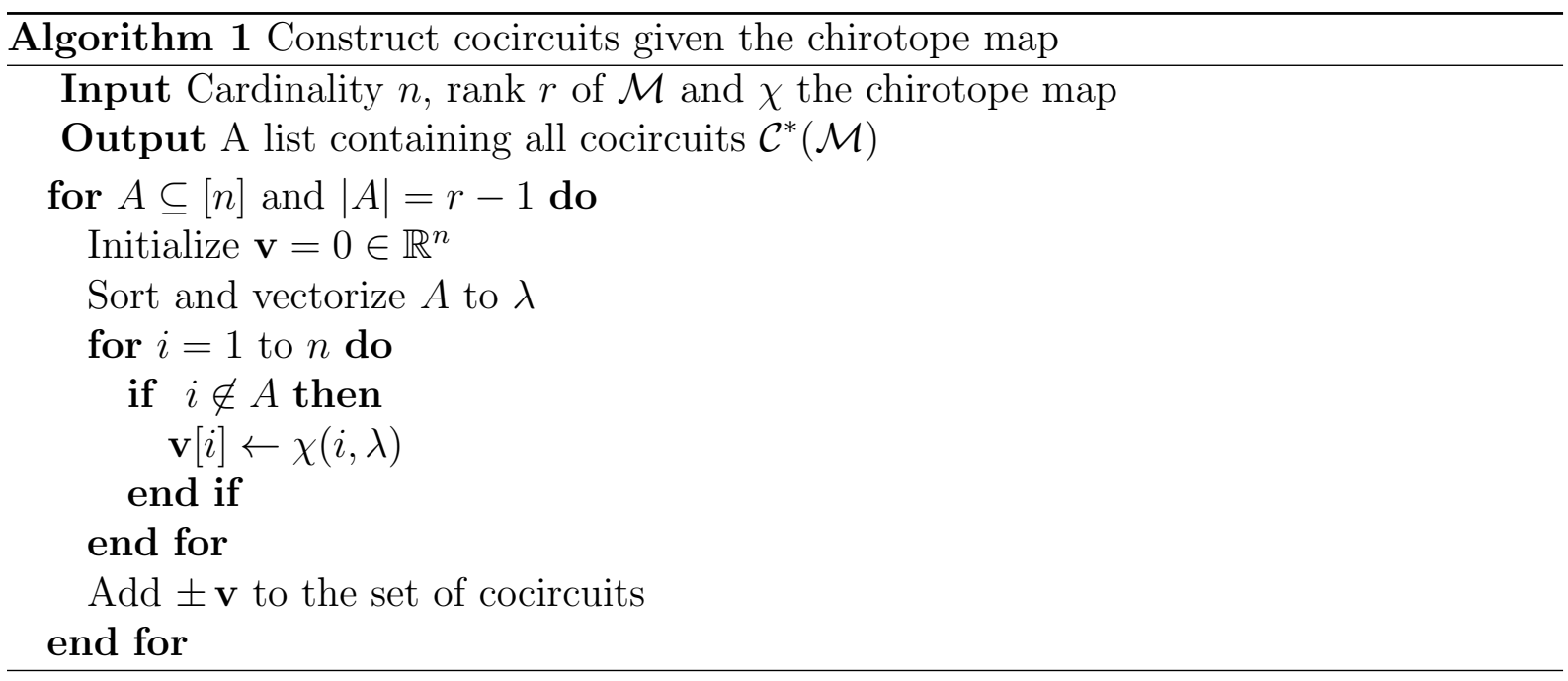

and compute their diameters. When $n=9, r=4,5$ the algorithm takes the longest to terminate. On average, each instance of an oriented matroid takes about 0.36 seconds to compute, resulting in around 38.7 days to complete the checking of all oriented matroids of cardinality nine and rank four.

We investigate other interesting questions such as whether the shortest path between two cocircuits on the same tope stays on the tope (see Section 5). Our code is available on Github. ${ }^{1}$ Based on our explicit computations, we derive the following theorem for small matroids, as promised in the introduction.

Theorem 15. Let $r \leqslant n \leqslant 9$ and $\mathcal{M} \in \operatorname{UOM}(n, r)$, then $\operatorname{diam}\left(G^{*}(\mathcal{M})\right)=n-r+2$. Moreover, if $X, Y \in \mathcal{C}^{*}(\mathcal{M})$ with $X \neq-Y$ and $n \leqslant 9$, then $d_{\mathcal{M}}(X, Y) \leqslant n-r+1$.

\subsection{Results in low rank}

As a next step, we explore Conjecture 1 in low rank. If $\mathcal{M} \in U O M(n, 2)$, then the cocircuit graph $G^{*}(\mathcal{M})$ is a cycle on $2 n$ vertices, so its diameter is $n=n-r+2$. Thus Conjecture 1 holds trivially when $r=2$. Now we move on to study uniform oriented matroids of rank three.

Theorem 16. Let $\mathcal{M} \in U O M(n, 3)$, then $\operatorname{diam}\left(G^{*}(\mathcal{M})\right)=n-r+2=n-1$.

Proof. Let $\mathcal{M} \in U O M(n, 3)$ and $X, Y \in \mathcal{C}^{*}(\mathcal{M})$. If $X=-Y$, then $d_{\mathcal{M}}(X, Y)=n-r+2$ by Lemma 5. If $\left|X^{0} \backslash Y^{0}\right|=1$, then $d_{\mathcal{M}}(X, Y) \leqslant n-r+1$ by Lemma 5 . So we only need to consider the case that $\left|X^{0} \backslash Y^{0}\right| \geqslant 2$. But $\left|X^{0}\right|=\left|Y^{0}\right|=r-1=2$, so this means $X^{0} \cap Y^{0}=\emptyset$.

Identify the elements of $E(\mathcal{M})$ with $\{1,2, \ldots, n\}$. Let $\mathcal{P}(\mathcal{M})$ be the Folkman-Lawrence representation of $\mathcal{M}$ with pseudospheres $\left\{s_{1}, \ldots, s_{n}\right\}$.

Without loss of generality we can assume $X^{0}=\{1,2\}$ and $Y^{0}=\{3,4\}$. Let $\mathcal{M}^{\prime}$ denote the restriction of $\mathcal{M}$ to $\{1,2,3,4\} \subseteq E$. The Folkman-Lawrence representation of $\mathcal{M}^{\prime}$ is

\footnotetext{
${ }^{1}$ https://github.com/zzy1995/OrientedMatroid
} 


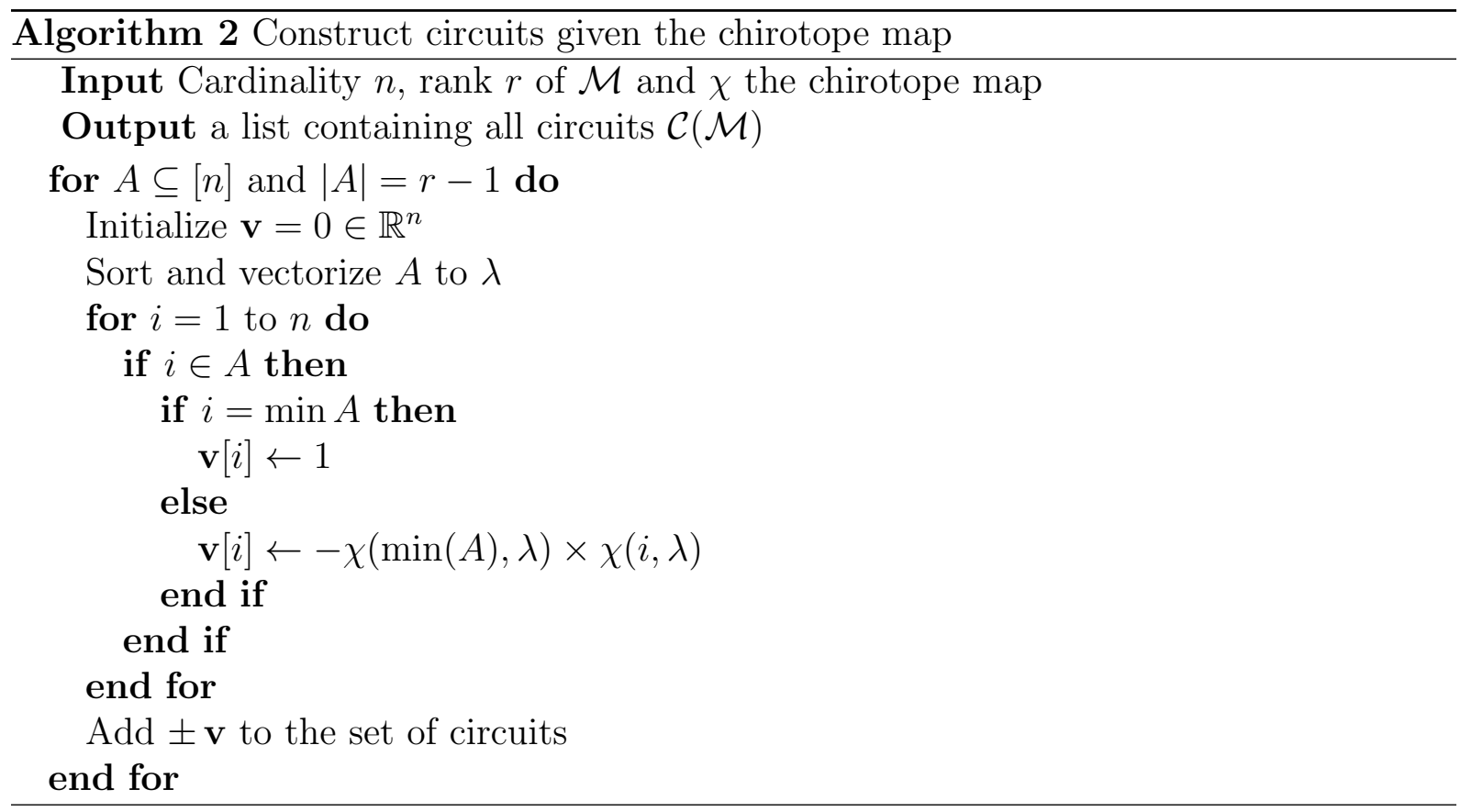

obtained from $\mathcal{P}(\mathcal{M})$ by removing $s_{i}$ for all $i>4$. Up to relabeling and reorientation, there is only one uniform oriented matroid of rank three on four elements. We can further assume $X_{3}=X_{4}=Y_{1}=Y_{2}=+$. In particular, there are cocircuits $W$ and $Z$ such that $W^{0}=\{1,3\}, Z^{0}=\{2,4\}$, and $W_{2}=W_{4}=Z_{1}=Z_{3}=+$. Consider the region, $D=s_{1}^{+} \cap s_{2}^{+} \cap s_{3}^{+} \cap s_{4}^{+} \subseteq \mathcal{P}(\mathcal{M})$. This is the quadrilateral region bounded by cocircuits $X, Y, Z$, and $W$ in Figure 4 .

We claim that for each $i>4$, the pseudosphere $s_{i}$ can intersect the boundary of $D$ in at most two points. Indeed, suppose $s_{i}$ intersects the boundary of $D$ at a point $p_{0} \in s_{j}$ for some $j \in\{1,2,3,4\}$. Because $\mathcal{M}$ is uniform, $p_{0} \notin\{X, Y, Z, W\}$, so $s_{j}$ is unique. Let $\varphi_{i}:[0,1] \rightarrow \mathcal{P}(\mathcal{M})$ be a parametrization of $s_{i}$. We can assume $\varphi_{i}(0)=p_{0}$ and $\varphi_{i}(t)$ passes into the interior of $D$ for sufficiently small $t>0$. Let $t_{1}$ be the next time when $\varphi_{i}\left(t_{1}\right)$ is on the boundary of $D$. Assume $\varphi_{i}\left(t_{1}\right) \in s_{k}$. Once again, $s_{k}$ is unique because $\mathcal{M}$ is uniform. Further, $k \neq j$ because otherwise $s_{j}$ would intersect $s_{i}$ in at least four points: $\varphi_{i}(0), \varphi_{i}\left(t_{1}\right)$, and their antipodes.

When $t>0$ is sufficiently small, $\varphi_{i}(t) \in s_{j}^{+} \cap s_{k}^{+}$. When $t>t_{1}$ and $t-t_{1}$ is sufficiently small, $\varphi_{i}(t) \in s_{j}^{+} \cap s_{k}^{-}$. By the definition of a pseudosphere arrangement, the image of $\varphi_{i}$ cannot cross back into $s_{k}^{+}$before it crosses into $s_{j}^{-}$. However, any other points where the image of $\varphi_{i}$ could intersect the boundary of $D$ lie in $s_{j}^{+} \cap s_{k}^{+}$. Thus $\varphi_{i}(0)$ and $\varphi_{i}\left(t_{1}\right)$ are the only points of intersection of $s_{i}$ with the boundary of $D$.

Now we consider two paths from $X$ to $Y$ in $G^{*}(\mathcal{M})$. The first path $P_{W}$ travels from $X$ to $W$ along $s_{1}$, then from $W$ to $Y$ along $s_{3}$. The second path $P_{Z}$ travels from $X$ to $Z$ along $s_{2}$, then from $Z$ to $Y$ along $s_{4}$. Let $\ell\left(P_{W}\right)$ and $\ell\left(P_{Z}\right)$ denote the lengths of these paths. Initially, in $\mathcal{M}^{\prime}, \ell\left(P_{W}\right)=\ell\left(P_{Z}\right)=2$.

For each $i>4$, the pseudosphere $s_{i}$ meets the boundary of $D$ in at most two points. 


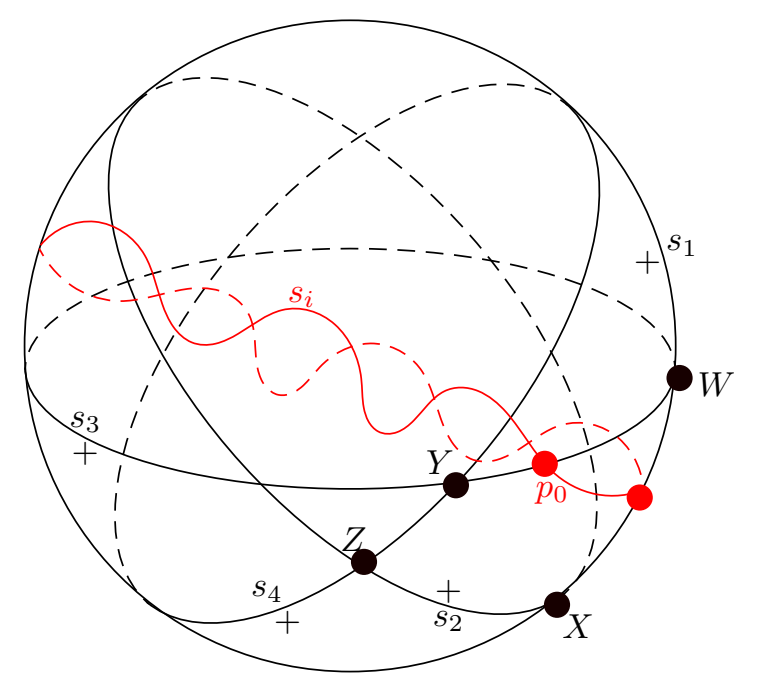

Figure 4: The unique rank-3 pseudosphere arrangement with four pseudolines.

This means $\ell\left(P_{W}\right)+\ell\left(P_{Z}\right)$ increases by at most two when we add $s_{i}$ back into $\mathcal{P}(\mathcal{M})$. Thus, in $\mathcal{M}$,

$$
\ell\left(P_{W}\right)+\ell\left(P_{Z}\right) \leqslant 4+2(n-4)=2 n-4 .
$$

By the pigeonhole principle, either $\ell\left(P_{W}\right) \leqslant n-2$ or $\ell\left(P_{Z}\right) \leqslant n-2$, so $d_{\mathcal{M}}(X, Y) \leqslant$ $n-2$.

Corollary 17. Let $r \geqslant 3$ and $\mathcal{M} \in U O M(n, r)$. If $X, Y \in \mathcal{C}^{*}(\mathcal{M})$ and $\left|X^{0} \backslash Y^{0}\right|=2$, then $d_{\mathcal{M}}(X, Y) \leqslant n-r+1$.

Proof. Let $A=X^{0} \cap Y^{0}$. Let $\left\{s_{e}: e \in E(\mathcal{M})\right\}$ be the pseudospheres in the FolkmanLawrence representation of $\mathcal{M}$ and let $S_{A}=\bigcap_{e \in A} s_{e}$. Because $\mathcal{M}$ is uniform, $|A|=r-3$ and hence $S_{A} \approx \mathbb{S}^{2}$ is the Folkman-Lawrence representation of the uniform oriented matroid $\mathcal{M} / A \in U O M(n-r+3,3)$.

Both $X$ and $Y$ are cocircuits on $S_{A}$ and clearly $X \neq-Y$, so by Theorem 16 ,

$$
d_{\mathcal{M}}(X, Y) \leqslant d_{\mathcal{M} / A}(X, Y) \leqslant(n-r+3)-2=n-r+1 .
$$

Recall that in the proof of Theorem 16 for oriented matroids of rank three, the two cocircuits we choose lie on four different hyperplanes, and they form a combinatorial square. Each additional hyperplane will intersect the square twice, which implies that one of the two paths will at most increase by one. Santos (personal communication) has pointed out that this cannot be directly extended to establish Conjecture 1 in rank four. For a realizable uniform oriented matroid of rank four, six hyperplanes will enclose a combinatorial cube. For concreteness, we can consider the cube with $-1 \leqslant x_{i} \leqslant 1$ for all $i=1,2,3$.

Figure 5 illustrates three edge-disjoint paths, colored red, green, and blue, from $(-1,-1,-1)$ to $(1,1,1)$. Here, $(-1,-1,-1)$ is the vertex incident to the three dotted 
edges, and $(1,1,1)$ is its polar opposite. The three images show slices of the cube by hyperplanes $x_{i}+x_{j}=\left(2-\varepsilon_{k}\right) x_{k}$ for all choices of $\{i, j, k\}=\{1,2,3\}$ and with $\varepsilon_{1}, \varepsilon_{2}$, and $\varepsilon_{3}$ all distinct. Each plane intersects two edges incident to $(-1,-1,-1)$ and two edges incident to $(1,1,1)$, and hence increases the total length of all three paths by at least four. If each of the remaining $n-6$ hyperplanes has one of the three illustrated types (with the $\varepsilon_{k}$ generic) then the total length of the red, blue, and green paths will be at least $4(n-6)+9$. If there are approximately $\frac{n-6}{3}$ hyperplanes of each type, then each of the red, green, and blue paths will have length at least $\left\lfloor\frac{4}{3} n\right\rfloor-5$.
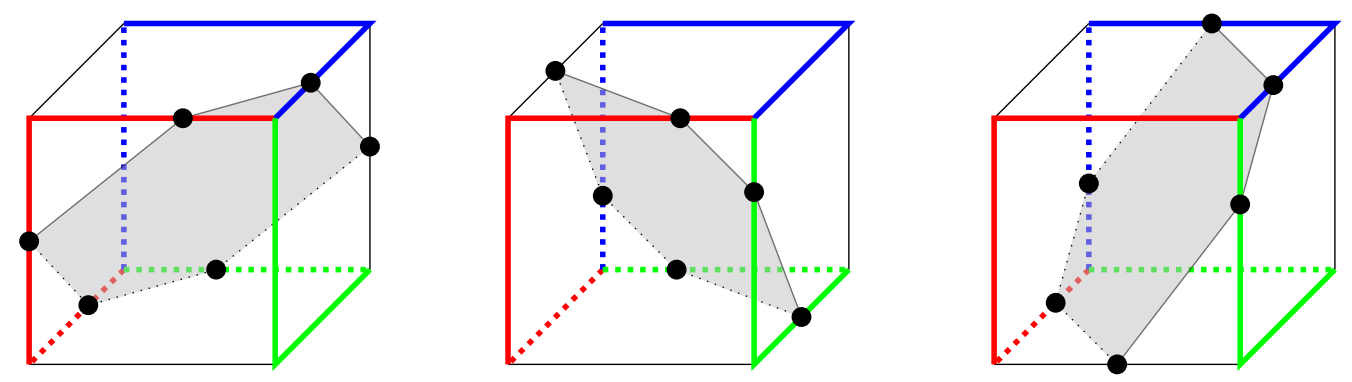

Figure 5: Hyperplanes $x_{i}+x_{j}=\left(2-\varepsilon_{k}\right) x_{k}$ slicing the \pm 1 cube for $\{i, j, k\}=\{1,2,3\}$.

\subsection{Results in low corank}

Recall that the corank of an oriented matroid of rank $r$ on $n$ elements is equal to $n-r$.

Lemma 18. Let $\mathcal{M} \in U O M(n, r)$ with $n-r=k$ for $k \geqslant 0$. Then

$$
\operatorname{diam}\left(G^{*}(\mathcal{M})\right) \leqslant \max \left\{\operatorname{diam}\left(G^{*}\left(\mathcal{M}^{\prime}\right)\right): \mathcal{M}^{\prime} \in U O M\left(r^{\prime}+k, r^{\prime}\right), 2 \leqslant r^{\prime} \leqslant k+2\right\} .
$$

Proof. Let $\mathcal{M}$ be a uniform oriented matroid of corank $k$, and let $X, Y \in \mathcal{C}^{*}(\mathcal{M})$ such that $\operatorname{diam}\left(G^{*}(\mathcal{M})\right)=d_{\mathcal{M}}(X, Y)$. If $Y=-X$, we are done, since by, Lemma 5 the diameter of any uniform oriented matroid of corank $k$ is at least $k+2$, and $d_{\mathcal{M}}(X,-X)=k+2$. So we assume that $Y \neq-X$.

Consider the contraction $\mathcal{M}^{\prime}=\mathcal{M} /\left(X^{0} \cap Y^{0}\right)$, and let $X^{\prime}$ and $Y^{\prime}$ be the images of $X$ and $Y$ under this contraction. Let $r^{\prime}=\operatorname{rank}\left(\mathcal{M}^{\prime}\right)$ and $n^{\prime}=\left|E\left(\mathcal{M}^{\prime}\right)\right|$. We know that $\mathcal{M}^{\prime}$ is uniform because $\mathcal{M}$ is. Note that $\left(X^{\prime}\right)^{0} \cap\left(Y^{\prime}\right)^{0}=\emptyset$ by construction, so $\operatorname{supp}\left(X^{\prime}\right) \cup$ $\operatorname{supp}\left(Y^{\prime}\right)=E\left(\mathcal{M}^{\prime}\right)$. In addition, since $\mathcal{M}^{\prime}$ is uniform, $\left|\operatorname{supp}\left(X^{\prime}\right)\right|=\left|\operatorname{supp}\left(Y^{\prime}\right)\right|=k+1$. This shows $\left|E\left(\mathcal{M}^{\prime}\right)\right| \leqslant 2(k+1)$. Further, $\operatorname{supp}\left(X^{\prime}\right) \neq \operatorname{supp}\left(Y^{\prime}\right)$ because $Y \neq-X$, so $\left|\operatorname{supp}\left(X^{\prime}\right) \cup \operatorname{supp}\left(Y^{\prime}\right)\right| \geqslant k+2$, which implies $2 \leqslant r^{\prime} \leqslant k+1$, as $\left|E\left(\mathcal{M}^{\prime}\right)\right|=r^{\prime}+k$.

Then, as $X^{\prime}, Y^{\prime} \in \mathcal{C}^{*}\left(\mathcal{M}^{\prime}\right)$ and $G^{*}\left(\mathcal{M}^{\prime}\right)$ is a subgraph of $G^{*}(\mathcal{M})$, we have that $\operatorname{diam}\left(G^{*}(\mathcal{M})\right)=d_{\mathcal{M}}(X, Y) \leqslant d_{\mathcal{M}^{\prime}}\left(X^{\prime}, Y^{\prime}\right) \leqslant \operatorname{diam}\left(G^{*}\left(\mathcal{M}^{\prime}\right)\right)$. Thus, we conclude that for every matroid $\mathcal{M}$ of corank $k$, there exists a matroid $\mathcal{M}^{\prime} \in U O M\left(r^{\prime}+k, r^{\prime}\right)$, where $2 \leqslant r^{\prime} \leqslant k+2$, such that $\operatorname{diam}\left(G^{*}(\mathcal{M})\right) \leqslant \operatorname{diam}\left(G^{*}\left(\mathcal{M}^{\prime}\right)\right)$.

Theorem 19. Let $\mathcal{M} \in \operatorname{UOM}(n, r)$ with $n-r \leqslant 4$. Then $\operatorname{diam}\left(G^{*}(\mathcal{M})\right)=n-r+2$. 
Proof. If $n-r \leqslant 3$ the theorem follows directly from Lemma 18 and Theorem 15 .

When $n-r=4$, by Lemma 18 we have $\Delta(r+4, r) \leqslant \max _{2 \leqslant r^{\prime} \leqslant 6}\left\{\Delta\left(r^{\prime}+4, r^{\prime}\right)\right\}$. However, by Theorem 15 , for $2 \leqslant r^{\prime} \leqslant 5, \max \left\{\Delta\left(r^{\prime}+4, r^{\prime}\right)\right\} \leqslant r^{\prime}+4-r^{\prime}+2=6$. So we only need to consider $\mathcal{M} \in U O M(10,6)$. Let $X, Y \in \mathcal{C}^{*}(\mathcal{M})$ be such that $\operatorname{diam}\left(G^{*}(\mathcal{M})\right)=d_{\mathcal{M}}(X, Y)$. If $Y=-X$, the result holds by Lemma 5. If $X^{0} \cap Y^{0} \neq \emptyset$, then as in Theorem 18, the contraction $\mathcal{M}^{\prime}=\mathcal{M} /\left(X^{0} \cap Y^{0}\right)$ satisfies $d_{\mathcal{M}}(X, Y) \leqslant \operatorname{diam}\left(\mathcal{M}^{\prime}\right)$. Since $\left|E\left(\mathcal{M}^{\prime}\right)\right| \leqslant 9$, the result holds by Theorem 15. So we may assume that $X^{0} \cap Y^{0}=\emptyset$.

Define $\mathcal{T}=X \circ Y$. Then, by Lemma 13 the graph $G(\mathcal{T})$ of $\mathcal{T}$ is isomorphic to the graph $G_{A}(\mathcal{A})$ of $\mathcal{A}$, where $\mathcal{A}$ is the abstract polytope on the covector of $\mathcal{T}$ with dimension 5 on 10 elements. However, by [1, Theorem 7.1] the diameter of $G_{A}(\mathcal{A})$ is 5 , implying that $d_{\mathcal{M}}(X, Y)=5$. Noting that $d_{\mathcal{M}}(X,-X)=6$, we conclude that $\operatorname{diam}\left(G^{*}(\mathcal{M})\right)=6$ which completes the proof.

Note that while the theorems about coranks in this subsection are for uniform oriented matroids, they are valid for general oriented matroids due to Lemma 4. Now we are ready to combine all the results in this section to prove Theorem 6 .

Proof. (of Theorem 6)

The proof of part (a) for small oriented matroids is in Theorem 15. The proof of part (b) for rank three oriented matroids is in Theorem 16. The proof of part (c) for oriented matroids of corank no more than four is in Theorem 19.

\section{An Improved Quadratic Diameter Bound}

Let $\mathcal{M}$ be an oriented matroid. Recall that a coline in $\mathcal{M}$ is a one-dimensional sphere in the Folkman-Lawrence representation of $\mathcal{M}$. Now we present an improved quadratic upper bound on $\Delta(n, r)$ for uniform oriented matroids, improving Theorem 2. In particular, our expression (2) is tight for rank three. Our proof relies on a modification of Finschi's proof $[12]$.

\section{Proof. (of Theorem 7)}

By Lemma 4, it suffices to consider the case that $\mathcal{M}$ is uniform. We prove the claim by induction on $\left|X^{0} \backslash Y^{0}\right|$. If $\left|X^{0} \backslash Y^{0}\right|=1$, then $d_{\mathcal{M}}(X, Y) \leqslant n-r+1$ by Lemma 5 . If $\left|X^{0} \backslash Y^{0}\right|=2$, then $d_{\mathcal{M}}(X, Y) \leqslant n-r+1$ by Corollary 17 .

Now we move on to the inductive step. Suppose $\left|X^{0} \backslash Y^{0}\right|=\ell \geqslant 3$. Pick any element $e \in Y^{0} \backslash X^{0}$, and consider the coline $U$, with $U^{0}=Y^{0} \backslash\{e\}$. Note that $\left|U^{0} \backslash X^{0}\right|=\ell-1$.

Now we look more carefully at the coline $U$, which is a cycle on $2(n-r+2)$ cocircuits. We distinguish $\ell$ pairs of these cocircuits. For each element $f \in X^{0} \backslash U^{0}$, there is a cocircuit $Z^{f}$ with $\left(Z^{f}\right)^{0}=U^{0} \cup\{f\}$. Because $\left|X^{0} \backslash U^{0}\right|=\ell$, there are $\ell$ such pairs of antipodal cocircuits, which we denote as $\pm Z^{1}, \ldots, \pm Z^{\ell}$ for simplicity.

The cocircuits $Y$ and $-Y$ are antipodal on $U$, and hence partition $U$ into two halves, each of which contains $n-r+1$ cocircuits. Assume without loss of generality that $Z^{1}, \ldots, Z^{\ell}$ all lie on one half of the coline (as it is partitioned by $Y$ and $-Y$ ), and further that $Z^{1}, \ldots, Z^{\ell}$ are ordered by their distance from $Y$, with $Z^{1}$ closest to $Y$ and $Z^{\ell}$ farthest. 
Because there are $(n-r+2)-(\ell+1)=n-r-\ell+1$ remaining pairs of antipodal circuits on $U$, and at most one element from each pair can lie on the arc from $Z^{1}$ to $-Z^{\ell}$ that contains $Y$, it follows that there exists a path of length at most $\left\lfloor\frac{n-r-\ell+1}{2}\right\rfloor+1$ from $Y$ to one of $Z^{1}$ or $-Z^{\ell}$ along $U$. For simplicity, let $Z$ denote whichever of $Z^{1}$ and $-Z^{\ell}$ is closer to $Y$ along $U$.

In summary, we have shown that there exists a cocircuit $Z$ whose distance to $Y$ is at most $\left\lfloor\frac{n-r-\ell+1}{2}\right\rfloor+1$ with $\left|X^{0} \backslash Z^{0}\right|=\ell-1$. Because $\ell-1 \neq 0$, we know $Z \neq-X$ as well. The result now follows by induction, and after reindexing with $k=\ell-1$ we have

$$
d_{\mathcal{M}}(X, Y) \leqslant n-r+1+\sum_{k=2}^{\left|X^{0} \backslash Y^{0}\right|-1}\left(\left\lfloor\frac{n-r-k}{2}\right\rfloor+1\right) .
$$

To get Eq. (3), note that $\left|X^{0} \backslash Y^{0}\right| \leqslant \min (r-1, n-r+1)$, because $\left|X^{0} \backslash Y^{0}\right| \leqslant\left|X^{0}\right|=$ $r-1$ and $\left|X^{0} \backslash Y^{0}\right| \leqslant\left|E \backslash Y^{0}\right|=n-r+1$. So, when $r \geqslant 4$ and $n-r \geqslant 2$,

$$
\operatorname{diam}\left(G^{*}(\mathcal{M})\right) \leqslant n-r+1+\sum_{k=2}^{\min (r-2, n-r)}\left(\left\lfloor\frac{n-r-k}{2}\right\rfloor+1\right) .
$$

\section{Similarities to the diameter of polytopes problem and two conjectures}

One could hope that $d_{\mathcal{M}}(X, Y) \leqslant n-r+1$ provided $X, Y \in \mathcal{C}^{*}(\mathcal{M})$ are not antipodal cocircuits. However, this is not the case. Matschke, Santos, and Weibel [22] built on the methodology of Santos's original non-Hirsch polytope [25] to construct a simple polytope $P_{20,40}$ of dimension 20 with 40 facets which has diameter 21 . Let $\mathcal{M}_{20,40}$ be the oriented matroid obtained by lifting $P_{20,40}$ into $\mathbb{R}^{21}$ and intersecting its hyperplane arrangement with the unit sphere. Since $P_{20,40}$ is simple, $\mathcal{M}_{20,40}$ is uniform, and one of its topes is $P_{20,40}$. We will show that the oriented matroid $\mathcal{M}_{20,40} \in U O M(40,21)$ has a pair of non-antipodal cocircuits $X$ and $Y$ such that $d_{\mathcal{M}_{20,40}}(X, Y) \geqslant 21=n-r+2$.

Proof. (of Proposition 8)

Let $X, Y$ be the pair of cocircuits that are of distance 21 in $P_{20,40}$. Let $E=\{1, \ldots, 40\}$. After reorientation and relabeling, we may assume that $X^{0}=\{1,2, \ldots, 20\}, X^{+}=$ $\{21, \ldots, 40\}$ and $Y^{0}=\{21, \ldots, 40\}, Y^{+}=\{1, \ldots, 20\}$.

Consider a shortest path, $\gamma$, from $X$ to $Y$ in $\mathcal{M}_{20,40}$. If each cocircuit on $\gamma$ belongs to the tope $P_{20,40}$, then its length is 21. So we may suppose instead that $\gamma$ contains a cocircuit $Z$ that does not belong to $P_{20,40}$. This means $Z^{-} \neq \emptyset$.

Recall the notion of a "basic transformation" from the proof of Lemma 5. Each edge in the cocircuit graph accounts for two basic transformations, which change some entry on a cocircuit from $+/-$ to 0 or from 0 to $+/-$.

Let $i \in Z^{-}$. If $X_{i}=+$ and $Y_{i}=0$, then walking from $X$ to $Y$ via $Z$ requires at least $20+19+3=42$ basic transformations. This is because each $j \in X^{0}$ requires one basic 
transformation to become an element of $Y^{+}$; each $j \in X^{+} \backslash\{i\}$ requires one basic transformation to become an element of $Y^{0}$, and $i \in X^{+}$requires two basic transformations to become an element of $Z^{-}$and one additional transformation to subsequently become an element of $Y^{0}$. Similarly, if $X_{i}=0$ and $Y_{i}=+$, then walking from $X$ to $Y$ via $Z$ also requires at least 42 basic transformations. This tells us $d_{\mathcal{M}_{20,40}}(X, Y) \geqslant 21=n-r+2$.

We now prove Theorem 9. Let $\mathcal{M}$ be a uniform oriented matroid. We say a path $X^{1}, X^{2}, \ldots, X^{k}$ in the cocircuit graph $G^{*}(\mathcal{M})$ stays on a tope $\mathcal{T}$ if each cocircuit $X^{i}$ is a vertex of $\mathcal{T}$.

Proof. (of Theorem 9) We used computers to look over all (chirotopes) oriented matroids with $n \leqslant 10$ computed by Finschi and Fukuda [13] (we used them already earlier in the paper). We used the Python package NetworkX [9] to find all shortest paths between a given pair of cocircuits and verify that one of the shortest paths between them is a crabbed path. We also checked when shortest paths stay on a common tope. We found that for all $\mathcal{M} \in U O M(n, r)$ with $n \leqslant 8$, there exists a crabbed path from $X$ to $Y$ whose length is no bigger than the length of any path from $X$ to $Y$ in the entire cocircuit graph $\mathcal{M}$. But we eventually found a smallest counterexample in our search. This is an oriented matroid with 9 elements in rank 4. The chirotope mapping of this counterexample is

$+++++++++++++++++++++++++++++++++--++++++$ $+++++++++-++--+++++-+---+----++-++-+-+++-+$ $--+--+++----+--++--++++----+--++--++---+-$ shown here ordered by reverse lexicographical order. That is, the first two and the last two signs in the list (shown in red there) are $\chi(1,2,3,4,5)=+, \chi(1,2,3,4,6)=$ ,$+ \chi(4,6,7,8,9)=+, \chi(5,6,7,8,9)=-$.

Cocircuit $X_{12}=(0,0,-,-,-,-,-,-, 0)$ and cocircuit $X_{37}=(0,-,-,-, 0,0,-,-,+)$ lie on the same tope. But as shown in Figure 6, the shortest path between $X_{12}$ and $X_{37}$ goes through cocircuits $X_{85}$ and $X_{79}$, where

$$
X_{85}=(+, 0,-,-, 0,-,-,-, 0), X_{79}=(+, 0,-,-, 0,0,-,-,+) .
$$

Note that $X_{85}^{+}=\{1\} \nsubseteq X_{12}^{+} \cup X_{37}^{+}$, and thus the shortest path is shorter than any crabbed path.

Next, we take a hyperplane arrangement that realizes the tope in Figure 6 as a polytope and add a tenth hyperplane that cuts through (among others) the edge between $X_{37}$ and $X_{79}, X_{85}$ and $X_{12}$, as shown in Figure 7. By lifting all these hyperplanes (see Figure 1 for intuition of what is happening, we go from three to four dimensions), we obtain the central hyperplane arrangement that yields a (realizable) uniform oriented matroid $\mathcal{M}^{\prime}$ of rank 4 with 10 elements. Below are the explicit equations of these ten hyperplanes of the arrangement:

$$
\begin{aligned}
& H_{0}:-8 x_{1}-15.99 x_{2}-9 x_{3}+160 z=0, \\
& H_{1}:-56 x_{1}+112 x_{2}-39 x_{3}+672 z=0, \\
& H_{2}: 56 x_{1}-112 x_{2}-39 x_{3}+448 z=0,
\end{aligned}
$$



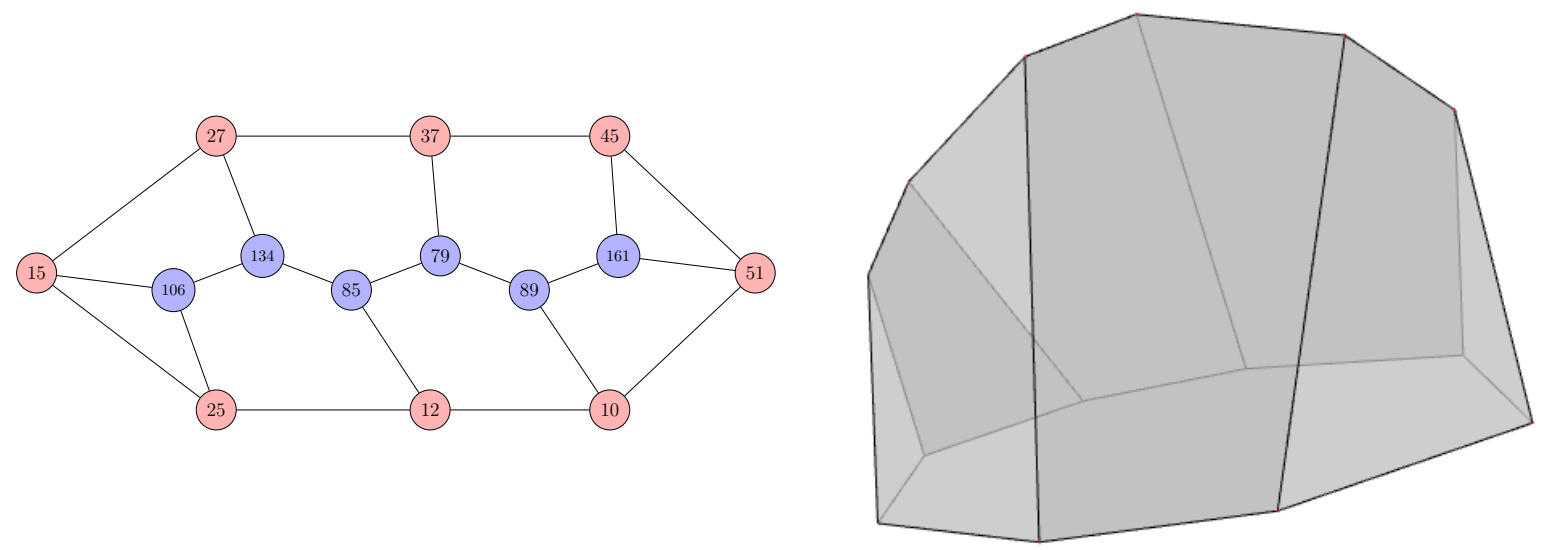

Figure 6: The subgraph induced by the tope containing $X_{12}$ and $X_{37}$ (left) and a realization of the tope as a 3-polytope (right).
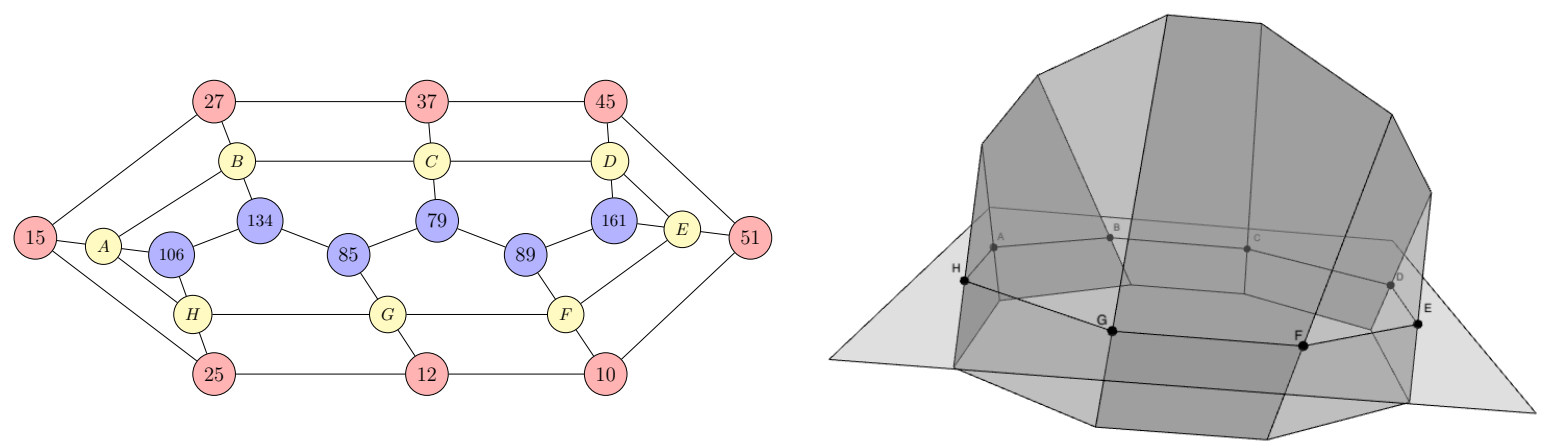

Figure 7: The subgraph after the tenth hyperplane is added, creating cocircuits $X_{A}^{\prime}, \ldots, X_{H}^{\prime}$ (left) and a realization of the tope and the hyperplane (right). The relevant shortest path is $X_{37}^{\prime} \rightarrow X_{C}^{\prime} \rightarrow X_{79}^{\prime} \rightarrow X_{85}^{\prime} \rightarrow X_{G}^{\prime}$.

$$
\begin{aligned}
& H_{3}: 8 x_{1}+16 x_{2}-9 x_{3}=0, \\
& H_{4}:-2 x_{2}-x_{3}+12 z=0, \\
& H_{5}: 280 x_{1}-31 x_{3}=0, \\
& H_{6}: x_{3}=0 \\
& H_{7}: 2 x_{2}-x_{3}+4 z=0, \\
& H_{8}:-280 x_{1}-31 x_{3}+3360 z=0, \\
& H_{9}: x_{1}+2 x_{2}+100 x_{3}-300 z=0 .
\end{aligned}
$$

After constructing the cocircuit graph of $\mathcal{M}^{\prime}$, we find that the path $X_{37}^{\prime} \rightarrow X_{C}^{\prime} \rightarrow$ $X_{79}^{\prime} \rightarrow X_{85}^{\prime} \rightarrow X_{G}^{\prime}$, going from $X_{37}^{\prime}$ to $X_{G}^{\prime}$, leaves the tope they share. Their common tope is composed by the red and yellow vertices in Figure 7 (these are points with indices 
A to $\mathrm{H}$ ). The path we proposed is shorter than any path from $X_{37}^{\prime}$ to $X_{G}^{\prime}$ staying on their common tope. This shows two cocircuits on a common tope while the shortest path between them leaves the tope. This completes the proof of the second part of the theorem.

Remark 20. For polytopes, $\mathrm{t}$ is natural to ask the following question: if two vertices lie on a common facet, does there exist a shortest path between them that stays within that facet? One can show that this property implies the non-revisiting path property [19], and therefore implies the (linear) Hirsch conjecture. The linear Hirsch conjecture was disproved by Santos, thus we know the polytope version of must be false starting in dimension 20. But Aviv Adler (personal communication) pointed out to us that already in three dimensions it is possible to have two vertices on a common facet while the shortest path between them leaves the facet. Our Theorem 9 demonstrates this fails for oriented matroids too and here we provided the smallest counterexample.

\section{Acknowledgements}

The first author was supported by the Tsinghua-Berkeley Shenzhen Institute. The second and the fourth author were supported by NSF grant DMS-1818969. The third author was supported by NSF grant DMS-1600048. And the fourth author was also supported by NSF HDR TRIPODS grant CCF-1934568. We are grateful for the comments and suggestions we received from anonymous referees as well as from Aviv Adler, Louis Billera, Lukas Finschi, Komei Fukuda, Kolja Knauer, Nati Linial, Francisco Santos, and Tamás Terlaky.

\section{References}

[1] I. Adler and G.B.D. Dantzig. Maximum diameter of abstract polytopes. Mathematical Programming Study, 1:20-40, 1974.

[2] E. Babson, L. Finschi, and K. Fukuda. Cocircuit graphs and efficient orientation reconstruction in oriented matroids. European J. Combin., 22(5):587-600, 2001. Combinatorial geometries (Luminy, 1999). https://doi.org/10.1006/eujc. 2001.0481.

[3] D. Bertsimas and J. Tsitsiklis. Introduction to Linear Optimization. Athena Scientific Series in Optimization and Neural Computation. 1997.

[4] A. Björner, M. Las Vergnas, B. Sturmfels, N. White, and G. M. Ziegler. Oriented Matroids, volume 46 of Encyclopedia of Mathematics and its Applications. Cambridge University Press, Cambridge, second edition, 1999. https://doi.org/10.1017/CB09780511586507.

[5] R. Cordovil and K. Fukuda. Oriented matroids and combinatorial manifolds. European Journal of Combinatorics, 14(1):9-15, 1993. https://doi.org/10.1006/eujc.1993.1002.

[6] R. Cordovil, K. Fukuda, and A. Guedes de Oliveira. On the cocircuit graph of an oriented matroid. Discrete and Computational Geometry, 24:257-266, 092000. https://doi.org/10.1007/s004540010031. 
[7] F. Criado and F. Santos. The maximum diameter of pure simplicial complexes and pseudo-manifolds. Discrete \&6 Computational Geometry, 58(3):643-649, Oct 2017. https://doi.org/10.1007/s00454-017-9888-5.

[8] F. Criado and F. Santos. Topological prismatoids and small simplicial spheres of large diameter. 2019. arXiv:1807.03030.

[9] NetworkX Developers. Networkx, September 2018. URL: https://networkx. github.io/documentation/networkx-2.2/index.html.

[10] F. Eisenbrand, N. Hähnle, A. Razborov, and T. Rothvoß. Diameter of polyhedra: Limits of abstraction. Math. Oper. Res., 35(4):786-794, 2010. https://doi.org/10.1287/moor.1100.0470.

[11] S. Felsner, R. Gómez, K. Knauer, J. J. Montellano-Ballesteros, and R. Strausz. Cubic time recognition of cocircuit graphs of uniform oriented matroids. European $J$. Combin., 32(1):60-66, 2011. https://doi.org/10.1016/j.ejc.2010.07.012.

[12] L. Finschi. A graph theoretical approach for reconstruction and generation of oriented matroids. PhD thesis, ETH Zürich, 2001. https://doi.org/10.3929/ethz-a-004255224.

[13] L. Finschi and K. Fukuda. Combinatorial generation of small point configurations and hyperplane arrangements. CCCG '01, pages 97-100, 2001.

[14] L. Finschi and K. Fukuda. Homepage of oriented matroids, November 2001. URL: http://www.om.math.ethz.ch/.

[15] J. Folkman and J. Lawrence. Oriented matroids. J. Combin. Theory Ser. B, 25(2):199-236, 1978. https://doi .org/10.1016/0095-8956(78)90039-4.

[16] K. Fukuda and T. Terlaky. Criss-cross methods: A fresh view on pivot algorithms. Mathematical Programming: Series A and B, Volume 79 Issue 1-3:369-395, October 1997.

[17] K. Fukuda and T. Terlaky. On the existence of a short admissible pivot sequence for feasibility and linear optimization problems. Pure Mathematics and Applications, vol 10(4):431-447, 1999.

[18] G. Kalai and D. J. Kleitman. A quasi-polynomial bound for the diameter of graphs of polyhedra. Bull. Amer. Math. Soc. (N.S.), 26(2):315-316, 1992. https://doi .org/10.1090/S0273-0979-1992-00285-9.

[19] V. Klee and P. Kleinschmidt. The $d$-step conjecture and its relatives. Math. Oper. Res., 12(4):718-755, 1987. https://doi.org/10.1287/moor.12.4.718.

[20] V. Klee and D. W. Walkup. The $d$-step conjecture for polyhedra of dimension $d<6$. Acta Math., 117:53-78, 1967. https://doi.org/10.1007/BF02395040.

[21] K. Knauer, J. Montellano-Ballesteros, and R. Strausz. A graph-theoretical axiomatization of oriented matroids. European Journal of Combinatorics, 35:388-391, 2014. Selected Papers of EuroComb'11. https://doi.org/10.1016/j.ejc.2013.06.016. 
[22] B. Matschke, F. Santos, and C. Weibel. The width of five-dimensional prismatoids. Proc. Lond. Math. Soc. (3), 110(3):647-672, 2015. https://doi.org/10.1112/plms/pdu064.

[23] J.J. Montellano-Ballesteros and R. Strausz. A characterization of cocircuit graphs of uniform oriented matroids. J. Combin. Theory Ser. B, 96(4):445-454, 2006. https://doi.org/10.1016/j.jctb.2005.09.008.

[24] J. Richter-Gebert. Testing orientability for matroids is NP-complete. Advances in Applied Mathematics, 23:78-90, 1999.

[25] F. Santos. A counterexample to the Hirsch conjecture. Ann. of Math. (2), 176(1):383412, 2012. https://doi.org/10.4007/annals.2012.176.1.7.

[26] F. Santos. Recent progress on the combinatorial diameter of polytopes and simplicial complexes. TOP, 21(3):426-460, Oct 2013. https://doi .org/:10.1007/s11750-013-0295-7.

[27] A. Schrijver. Theory of Linear and Integer Programming. John Wiley and Sons, Inc. New York, NY, USA, 1986.

[28] N. Sukegawa. An asymptotically improved upper bound on the diameter of polyhedra. Discret. Comput. Geom., 62(3):690-699, 2019. https://doi .org/10.1007/s00454-018-0016-y.

[29] G. M. Ziegler. Lectures on polytopes, volume 152 of Graduate Texts in Mathematics. Springer-Verlag, New York, 1995. https://doi.org/10.1007/978-1-4613-8431-1. 